AEI-2012-081

\title{
TOWARDS METRIC-LIKE HIGHER-SPIN GAUGE THEORIES IN THREE DIMENSIONS
}

\author{
A. Campoleoni, S. Fredenhagen, S. Pfenninger and S. Theisen \\ Max-Planck-Institut für Gravitationsphysik \\ Albert-Einstein-Institut \\ Am Mühlenberg 1 \\ 14476 Golm, GERMANY \\ andrea.campoleoni@aei.mpg.de,stefan.fredenhagen@aei.mpg.de, \\ stefan.pfenninger@aei.mpg.de,stefan.theisen@aei.mpg.de
}

\begin{abstract}
We consider the coupling of a symmetric spin-3 gauge field $\varphi_{\mu \nu \rho}$ to three-dimensional gravity in a second order metric-like formulation. The action that corresponds to an $S L(3, \mathbb{R}) \times S L(3, \mathbb{R})$ Chern-Simons theory in the frame-like formulation is identified to quadratic order in the spin-3 field. We apply our result to compute corrections to the area law for higher-spin black holes using Wald's entropy formula.
\end{abstract}




\section{Contents}

1 Introduction and overview 2

2 Minimal coupling of higher spins to gravity $\quad 5$

2.1 General quadratic coupling for a spin-3 field . . . . . . . . . . . . . . . 6

2.2 Relation with the Chern-Simons action . . . . . . . . . . . . . . 9

2.3 Algebra of gauge transformations . . . . . . . . . . . . . . . . . 10

3 Mapping frame- to metric-like formulation $\quad 13$

3.1 Elimination of spin connections . . . . . . . . . . . . . . 13

3.2 Relations between gauge parameters . . . . . . . . . . . . . . 15

3.3 Transformations of metric-like fields . . . . . . . . . . . . . . . 16

4 Wald entropy for higher-spin black holes $\quad 17$

$\begin{array}{llr}5 & \text { Discussion } & 20\end{array}$

$\begin{array}{ll}\text { A Conventions } & 22\end{array}$

B Spin-3 transformation of the metric $\quad 23$

C Tensors of arbitrary rank $\quad 24$

\section{Introduction and overview}

By now much is known about the structure of interacting field theories involving particles of spin greater than two. In particular, Vasiliev proposed a set of non-linear equations of motion that describe the interactions of an infinite tower of gauge fields of increasing spin on (A)dS backgrounds [1] (see [2] for a review). This result rests upon a description of the dynamics that mimics the frame approach to gravity: the degrees of freedom are encoded in a set of differential forms taking values in an infinite-dimensional extension of the Lorentz algebra, and the field equations are manifestly invariant under diffeomorphisms. Alternative approaches to higher-spin interactions are also actively investigated in order to look for generalisations of Vasiliev's construction or to make more transparent various features of the interactions (see e.g. [3-5] for reviews). For instance, one can follow the path of the metric formulation of gravity and encode the degrees of freedom of a spin- $s$ 
particle in a symmetric tensor of rank $s$. The advantage with respect to Vasiliev's strategy is the simplification of the field content; the price to pay is, at present, the lack of an organising principle for the non-linearities required by a consistent theory. To unravel this puzzle one can begin by building perturbatively the first interaction vertices; this has led, for instance, to a classification of cubic vertices for arbitrary massless particles in both Minkowski and (A)dS backgrounds of dimension $D \geq 4[6-10] .{ }^{1}$ On the other hand, a complete metric-like reformulation of Vasiliev's equations is not known, while the existence of other models that are consistent beyond the cubic order is still controversial (see e.g. $[12-14,11,15])$.

In spite of closely related goals, the frame- and metric-like formulations have evolved rather independently. For few exceptions see e.g. [16-18] and refs. therein. With both approaches having their own advantages and drawbacks, an exchange of ideas is nonetheless expected to shed light on both sides. The goal of this paper is to start to establish a firm connection between them in three space-time dimensions, where higher-spin gauge theories take a remarkably simple form compared to their higher-dimensional counterparts. We focus on the gravitational coupling of a symmetric tensor of rank 3 . In the frame-like language this is described by a $S L(3, \mathbb{R}) \times S L(3, \mathbb{R})$ Chern-Simons $(\mathrm{CS})$ theory when a negative cosmological constant is present (see e.g. [19] and the previous works [20,21]). In appendix $\mathrm{C}$ we will add a few comments on the generalisation to $S L(N, \mathbb{R}) \times S L(N, \mathbb{R})$ CS theories, which contain fields of spin $2,3, \ldots, N$.

The frame-like theory is well understood, with and without cosmological constant: one has to complement the gravity dreibein and spin connection with two one-forms which play a similar role for the spin-3 field. The gauge connections can then be packed into two $\operatorname{sl}(3, \mathbb{R})$-valued forms $(\mathcal{A}=1, \ldots, 8$ and $a, b=0,1,2)$

$$
\begin{gathered}
e=e_{\mu}{ }^{\mathcal{A}} J_{\mathcal{A}} d x^{\mu}=\left(e_{\mu}{ }^{a} J_{a}+e_{\mu}{ }^{a b} T_{a b}\right) d x^{\mu}, \\
\omega=\omega_{\mu}^{\mathcal{A}} J_{\mathcal{A}} d x^{\mu}=\left(\omega_{\mu}{ }^{a} J_{a}+\omega_{\mu}{ }^{a b} T_{a b}\right) d x^{\mu},
\end{gathered}
$$

where $J_{\mathcal{A}}$ denotes the full set of $\operatorname{sl}(3, \mathbb{R})$ generators. The gravity dreibein $e_{\mu}{ }^{a}$ and spin connection $\omega_{\mu}{ }^{a}$ are associated with the generators $J_{a}$ of the principally embedded $s o(2,1) \simeq s l(2, \mathbb{R}) \hookrightarrow \operatorname{sl}(3, \mathbb{R})$. The remaining five generators $T_{a b}\left(\right.$ with $\left.T_{[a b]}=\eta^{a b} T_{a b}=0\right)$ are associated to the spin-3 "vielbein" and "spin connection". One can then consider the action $^{2}$

$$
I=\frac{1}{16 \pi G} \int \operatorname{tr}\left(e \wedge R+\frac{1}{3 \ell^{2}} e \wedge e \wedge e\right), \quad \text { with } \quad R=d \omega+\omega \wedge \omega .
$$

The trace is in the fundamental of $\operatorname{sl}(3, \mathbb{R}), G$ is Newton's constant and $\ell$ the AdS radius.

\footnotetext{
${ }^{1}$ The classification of cubic interactions for arbitrary fields is discussed in a frame-like language in [11].

${ }^{2}$ For $\ell^{2}>0$ (corresponding to a negative cosmological constant) one can rewrite (1.2) as the difference of two $\operatorname{sl}(3, \mathbb{R})$ CS actions. A cosmological constant is however not necessary in $D=3$, and for $\ell^{2} \leq 0$ one can interpret (1.2) as a CS action as well (see e.g. [22] for more details).
} 
A first step towards the identification of the metric-like counterpart of (1.2) was taken in [19], where the metric and the spin-3 field were expressed in terms of the connection one-forms (1.1) as

$$
g=\frac{1}{2} \operatorname{tr}\left(e_{\mu} e_{\nu}\right) d x^{\mu} d x^{\nu}, \quad \varphi=\frac{1}{6} \operatorname{tr}\left(e_{\mu} e_{\nu} e_{\rho}\right) d x^{\mu} d x^{\nu} d x^{\rho} .
$$

The justification for (1.3) is that the action (1.2) is invariant under the transformations

$$
\begin{aligned}
& \delta e=d \xi+[\omega, \xi]+[e, \Lambda], \\
& \delta \omega=d \Lambda+[\omega, \Lambda]+\frac{1}{\ell^{2}}[e, \xi],
\end{aligned}
$$

generated by $\operatorname{sl}(3, \mathbb{R})$-valued parameters $\xi$ and $\Lambda$. Those generated by $\Lambda$ include and generalise local Lorentz transformations: therefore metric-like fields should be invariant under them, and this is guaranteed by (1.3) (see also [23] for a discussion of the $\operatorname{sl}(N, \mathbb{R})$ case). The transformations with parameters $\xi$ should then give rise to the transformations of $g$ and $\varphi$ under diffeomorphisms and a suitable deformation of the linearised Fronsdal gauge symmetry [24].

In the present paper we verify these statements up to the quadratic order in the spin-3 field, while keeping all non-linearities in the metric. In particular, we show that the action (1.2) can be rewritten in terms of the metric-like fields (1.3) as

$$
\begin{aligned}
I & =\int \frac{d^{3} x \sqrt{-g}}{16 \pi G}\left\{\left(R+\frac{2}{\ell^{2}}\right)+\varphi^{\mu \nu \rho}\left(\mathcal{F}_{\mu \nu \rho}-\frac{3}{2} g_{(\mu \nu} \mathcal{F}_{\rho)}\right)-\frac{3}{2} R \varphi_{\mu \nu \rho} \varphi^{\mu \nu \rho}\right. \\
& \left.+\frac{9}{4} R_{\rho \sigma}\left(2 \varphi_{\mu \nu}^{\rho} \varphi^{\sigma \mu \nu}-\varphi^{\rho} \varphi^{\sigma}\right)-\frac{1}{\ell^{2}}\left(6 \varphi_{\mu \nu \rho} \varphi^{\mu \nu \rho}-9 \varphi_{\mu} \varphi^{\mu}\right)\right\}+\mathcal{O}\left(\varphi^{4}\right),
\end{aligned}
$$

where $R_{\mu \nu}$ is the Ricci tensor, $\nabla$ is the Levi-Civita connection for the metric defined in (1.3) and $\mathcal{F}_{\mu \nu \rho}$ denotes the covariantised Fronsdal tensor

$$
\mathcal{F}_{\mu \nu \rho}=\square \varphi_{\mu \nu \rho}-\frac{3}{2}\left(\nabla^{\lambda} \nabla_{(\mu} \varphi_{\nu \rho) \lambda}+\nabla_{(\mu} \nabla^{\lambda} \varphi_{\nu \rho) \lambda}\right)+3 \nabla_{(\mu} \nabla_{\nu} \varphi_{\rho)} .
$$

We also have defined $\varphi_{\mu} \equiv \varphi_{\mu \lambda}^{\lambda}$ and, likewise, $\mathcal{F}_{\mu}$ is the trace of the Fronsdal tensor. Indices between parentheses are meant to be symmetrised, and dividing by the number of terms that are needed for the symmetrisation is understood. ${ }^{3}$ We also show that the action (1.5) - while manifestly diffeomorphism invariant - is invariant under the gauge transformations

$$
\begin{aligned}
\delta \varphi_{\mu \nu \rho} & =3 \nabla_{(\mu} \xi_{\nu \rho)}+\mathcal{O}\left(\varphi^{2}\right) \\
\delta g_{\mu \nu}= & 12 \xi^{\rho \sigma}\left\{\nabla_{\rho} \varphi_{\mu \nu \sigma}-2 \nabla_{(\mu} \varphi_{\nu) \rho \sigma}+2 g_{\rho(\mu \mid}\left[\nabla \cdot \varphi_{\mid \nu) \sigma}-\nabla_{\sigma} \varphi_{\mid \nu)}-\nabla_{\mid \nu)} \varphi_{\sigma}\right]\right. \\
& \left.+\frac{1}{2} g_{\rho \mu} g_{\sigma \nu} \nabla \cdot \varphi-g_{\mu \nu}\left[\nabla \cdot \varphi_{\rho \sigma}-2 \nabla_{\rho} \varphi_{\sigma}\right]\right\}+\mathcal{O}\left(\varphi^{3}\right)
\end{aligned}
$$

\footnotetext{
${ }^{3}$ Note that this and several other conventions used in the present paper differ from those of [19].
} 
generated by a traceless $\xi^{\rho \sigma}$. It thus preserves the same amount of gauge symmetry as the sum of the linearised Einstein-Hilbert and Fronsdal actions.

Our results give further support to the interpretation of $S L(N, \mathbb{R}) \times S L(N, \mathbb{R})$ CS theories as higher-spin gauge theories, but at the present stage the metric-like action (1.5) is certainly more involved than its frame-like counterpart (1.2). The simplicity of the frame-like action has various advantages: for instance, it allowed to compute the asymptotic symmetries of $(1.2)$ on $A d S_{3}$ spaces $[25,19,26,23]$. The appearance of nonlinear $\mathcal{W}$-algebras then led to a duality conjecture between a class of higher-spin theories with matter couplings [27] and $\mathcal{W}_{N}$ minimal models [28]; see [29] for a review. Another interesting result was the identification of solutions of the field equations - flat connections - that generalise the BTZ black hole [30-36]. On the other hand, there are aspects of higher-spin theories, such as gravitational interactions, which might be easier to deal with in a metric-like theory. As an example, in this paper we use the action (1.5) to compute the entropy of higher-spin black holes using Wald's formula [37]. In a cylindrical coordinate system $(\rho, t, \theta)$, for a non-rotating black hole with a horizon at $\rho=\rho_{h}$ we find

$$
S=\left.\frac{A}{4 G}\left\{1-\frac{3}{2} \frac{\left(\varphi_{\theta \theta \theta}\right)^{2}}{\left(g_{\theta \theta}\right)^{3}}+\mathcal{O}\left(\varphi^{4}\right)\right\}\right|_{\rho=\rho_{h}},
$$

where $A$ denotes the length of the horizon.

The paper is organised as follows: to arrive at (1.5), we consider in sec. 2.1 the most general action quadratic in $\varphi$, that contains the minimal coupling to gravity of Fronsdal's action. In sec. 2.2 we fix the free parameters by requiring that its field equations are solved by the class of asymptotically $A d S_{3}$ extrema of (1.2) which we constructed in [19]. In sec. 2.3 we comment on the algebra generated by the gauge transformations (1.7). A generalisation to spin-s fields is relegated to Appendix C. In sec. 3 we rederive the result of sec. 2.2 by a direct elimination of the spin connections $\omega_{\mu}{ }^{a}$ and $\omega_{\mu}{ }^{a b}$ from (1.2), and we discuss the map between frame- and metric-like gauge transformations. Further details are presented in Appendix B. In sec. 4 we turn to applications of (1.5): we derive (1.8) and we compare our result with the proposal of $[32,33]$. We close with a discussion of possible future directions. Our conventions are collected in Appendix A.

\section{Minimal coupling of higher spins to gravity}

In this section we consider actions that contain the minimal coupling to gravity of Fronsdal's Lagrangian [24], but we also allow additional terms quadratic in $\varphi$ which are manifestly diffeomorphism invariant and with at most two derivatives. The frame-like action (1.2) is indeed diffeomorphism invariant, its linearisation reduces to Fronsdal's action [19] and on shell $\omega$ can be rewritten in terms of $e$ and its first derivative. We show that when $D=3$ all actions of this type are also invariant at the lowest order in $\varphi$ under a deformation of the linearised Fronsdal gauge symmetry and are actually related by field 
redefinitions. We eventually select the point in the parameter space of field redefinitions that corresponds to the action (1.2) through the map (1.3).

\subsection{General quadratic coupling for a spin-3 field}

The free propagation of a spin-3 particle in a Minkowski background of dimension $D \geq 4$ can be described by the Fronsdal equations [24]

$$
\mathcal{F}_{\mu \nu \rho} \equiv \square \varphi_{\mu \nu \rho}-3 \partial^{\lambda} \partial_{(\mu} \varphi_{\nu \rho) \lambda}+3 \partial_{(\mu} \partial_{\nu} \varphi_{\rho)}=0
$$

They can be derived from an action which is left invariant by the gauge transformations

$$
\delta \varphi_{\mu \nu \rho}=3 \partial_{(\mu} \xi_{\nu \rho)}, \quad \text { with } \quad \xi_{\lambda}^{\lambda}=0
$$

In $D \geq 4$ this guarantees the propagation of the correct number ${ }^{4}$ of d.o.f., while in $D=3$ it implies that there is no local dynamics associated to (2.1). In order to couple this system to gravity it is natural to try minimal coupling, i.e. the substitutions $\eta \rightarrow g$ and $\partial \rightarrow \nabla$, where $g$ is the space-time metric and $\nabla$ is the Levi-Civita connection. However, a consistent coupling must preserve all gauge symmetries of the linearised theory, while the covariantised Fronsdal tensor ${ }^{5}$

$$
\mathcal{F}_{\mu \nu \rho}=\square \varphi_{\mu \nu \rho}-\frac{3}{2}\left(\nabla^{\lambda} \nabla_{(\mu} \varphi_{\nu \rho) \lambda}+\nabla_{(\mu} \nabla^{\lambda} \varphi_{\nu \rho) \lambda}\right)+3 \nabla_{(\mu} \nabla_{\nu} \varphi_{\rho)}
$$

transforms under

$$
\delta \varphi_{\mu \nu \rho}=3 \nabla_{(\mu} \xi_{\nu \rho)}, \quad \text { with } \quad \xi_{\lambda}^{\lambda}=0
$$

as

$$
\begin{aligned}
\delta \mathcal{F}_{\mu \nu \rho}= & -6 \xi^{\lambda \sigma} \nabla_{(\mu \mid} R_{\lambda \mid \nu \rho) \sigma}-9 R_{\lambda(\mu \nu \mid \sigma} \nabla_{\mid \rho)} \xi^{\lambda \sigma}+6 R_{\lambda(\mu \nu \mid \sigma} \nabla^{\lambda} \xi_{\mid \rho)} \sigma \\
& -6 \xi_{(\mu \mid}^{\lambda} \nabla_{\lambda} R_{\mid \nu \rho)}+\frac{3}{2} R_{\lambda(\mu \mid} \nabla^{\lambda} \xi_{\mid \nu \rho)}-9 R_{\lambda(\mu} \nabla_{\nu} \xi_{\rho)}{ }^{\lambda}
\end{aligned}
$$

This equation lies at the heart of the Aragone-Deser argument against higher-spin interactions [38]: when $D \geq 4$ one cannot cancel the contributions in the Riemann tensor with a $\xi$-dependent gauge transformation of the metric, and even adding to the action non-minimal terms of the form $R_{\ldots} \varphi \ldots \varphi$.. does not improve the situation. In general one can overcome this problem in two ways; Fradkin and Vasiliev showed in [39] that in the presence of a cosmological constant one can cancel (2.5) by adding higher-derivative contributions to the action (see also $[40,11]$ ). This solution preserves the invariance under diffeomorphisms and eventually led to the Vasiliev equations, that indeed require a

\footnotetext{
${ }^{4}$ namely $\frac{1}{3 !}(D-3)(D-2)(D+2)$

${ }^{5}$ This definition assumes a conventional choice for the ordering of covariant derivatives. In the following we will consider curvature terms as well, so that there is no lack of generality in resolving the ambiguity in a convenient way.
} 
non-zero cosmological constant and are manifestly diffeomorphism invariant. As an alternative, one can abandon the minimal coupling and consider also the gravitational field as a fluctuation around a fixed background; this path allowed to identify non-trivial interactions even in flat space [6-10], but now diffeomorphisms (or a proper deformation thereof) have to be recovered order by order in the metric fluctuation.

When $D=3$ the solution is much simpler: the Weyl tensor vanishes, so that the dangerous terms are actually proportional to the Ricci tensor and can be canceled by a $\xi$-dependent transformation of the metric (even in flat space). Let us thus consider the action

$$
I=\frac{1}{16 \pi G} \int d^{3} x \sqrt{-g}\left(\mathcal{L}_{E H}+\mathcal{L}_{F}\right)
$$

where $\mathcal{L}_{E H}$ denotes the Einstein-Hilbert Lagrangian,

$$
\mathcal{L}_{E H}=R+\frac{2}{\ell^{2}}
$$

while

$$
\begin{aligned}
\mathcal{L}_{F} & =\varphi^{\mu \nu \rho}\left(\mathcal{F}_{\mu \nu \rho}-\frac{3}{2} g_{(\mu \nu} \mathcal{F}_{\rho)}\right)+\frac{1}{\ell^{2}}\left(m_{1} \varphi_{\mu \nu \rho} \varphi^{\mu \nu \rho}+m_{2} \varphi_{\mu} \varphi^{\mu}\right) \\
& +3 R_{\rho \sigma}\left(k_{1} \varphi_{\mu \nu}^{\rho} \varphi^{\sigma \mu \nu}+k_{2} \varphi_{\mu}^{\rho \sigma} \varphi^{\mu}+k_{3} \varphi^{\rho} \varphi^{\sigma}\right) \\
& +3 R\left(k_{4} \varphi_{\mu \nu \rho} \varphi^{\mu \nu \rho}+k_{5} \varphi_{\mu} \varphi^{\mu}\right)+\mathcal{O}\left(\varphi^{4}\right) .
\end{aligned}
$$

This is the most general Lagrangian quadratic in $\varphi$ that reduces to the Fronsdal one upon linearisation, is manifestly diffeomorphism invariant and contains at most two derivatives. As already recalled, the restriction on the number of derivatives is dictated by our goal to identify the metric-like counterpart of the frame-like action (1.2). On dimensional grounds one could also add terms in $\ell^{-1}$ and a single covariant derivative, but the only candidate is $\ell^{-1} \nabla \cdot \varphi_{\lambda}^{\lambda}$, a total derivative. Furthermore, higher-order corrections are at least quartic in $\varphi$, as one cannot build a scalar with the inverse metric, three spin-3 fields and two derivatives. Cubic contributions proportional to $\ell^{-1}$ and with a single derivative would be available, but they are not needed for the gauge invariance, and they cannot be generated by the elimination of $\omega$ from the action (1.2).

Under covariantised gauge transformations (2.4), the Lagrangian varies as

$$
\delta\left(\sqrt{-g}\left(\mathcal{L}_{E H}+\mathcal{L}_{F}\right)\right)=\left(\frac{\delta\left(\sqrt{-g} \mathcal{L}_{E H}\right)}{\delta g_{\mu \nu}} \delta g_{\mu \nu}+\frac{\delta\left(\sqrt{-g} \mathcal{L}_{F}\right)}{\delta \varphi_{\mu \nu \rho}} \delta \varphi_{\mu \nu \rho}\right)+\frac{\delta\left(\sqrt{-g} \mathcal{L}_{F}\right)}{\delta g_{\mu \nu}} \delta g_{\mu \nu} .
$$

The terms in parentheses could cancel, up to total derivatives, if the metric transforms with a gauge transformation linear in $\varphi$ and $\xi$. This is possible for any choice of the 
coefficients $k_{i}$, while the "mass" coefficients in (2.8) have to satisfy ${ }^{6}$

$$
m_{1}=6\left(k_{1}+3 k_{4}-1\right), \quad m_{2}=6\left(k_{2}+k_{3}+3 k_{5}+\frac{9}{4}\right) .
$$

One can indeed check that when (2.10) holds, the action (2.6) is invariant, at lowest order in $\varphi$, under (2.4) and under the simultaneous transformation

$$
\begin{aligned}
\delta g_{\mu \nu}= & \left\{2 k_{2} \varphi_{\mu \nu \rho} \nabla \cdot \xi^{\rho}+a\left(2 k_{1}+5\right) \varphi_{\rho \sigma(\mu} \nabla_{\nu)} \xi^{\rho \sigma}+2\left(2 k_{1}-3\right) \varphi_{\rho \sigma(\mu} \nabla^{\rho} \xi_{\nu)}{ }^{\sigma}\right. \\
& +\left(4 k_{3}+3\right) \varphi_{(\mu} \nabla \cdot \xi_{\nu)}+2 b\left(k_{2}+4\right) \varphi_{\rho} \nabla_{(\mu} \xi_{\nu)} \rho+k_{2} \varphi_{\rho} \nabla^{\rho} \xi_{\mu \nu} \\
& +4 \xi^{\rho \sigma} \nabla_{\rho} \varphi_{\mu \nu \sigma}+(a-1)\left(2 k_{1}+5\right) \xi^{\rho \sigma} \nabla_{(\mu} \varphi_{\nu) \rho \sigma}+8 \xi^{\rho}{ }_{(\mu} \nabla \cdot \varphi_{\nu) \rho} \\
& -8 \xi_{\rho(\mu} \nabla^{\rho} \varphi_{\nu)}+2(b-1)\left(k_{2}+4\right) \xi^{\rho}{ }_{(\mu} \nabla_{\nu)} \varphi_{\rho}+2 \xi_{\mu \nu} \nabla \cdot \varphi \\
& -g_{\mu \nu}\left[3\left(2 k_{1}+4 k_{4}-1\right) \varphi_{\rho \sigma \lambda} \nabla^{\rho} \xi^{\sigma \lambda}+4 \xi^{\rho \sigma} \nabla \cdot \varphi_{\rho \sigma}\right. \\
& \left.\left.+\left(4 k_{2}+4 k_{3}+8 k_{5}+3\right) \varphi_{\rho} \nabla \cdot \xi^{\rho}-8 \xi^{\rho \sigma} \nabla_{\rho} \varphi_{\sigma}\right]\right\} .
\end{aligned}
$$

The two parameters $a$ and $b$ are undetermined as they parameterise field dependent diffeomorphisms.

The last term in (2.9) does not vanish and higher-order corrections to both the action and the gauge transformations are needed to preserve the gauge symmetry. In secs. 2.2 and 2.3 we shall give further arguments for this.

We found that the coefficients $k_{i}$ are free, but this does not mean that we have a five-parameter family of interacting theories. Actually, one can remove all $R_{\ldots} \varphi \ldots \varphi \ldots$ contributions with the field redefinition

$$
\begin{aligned}
g_{\mu \nu}^{(\text {new })}=g_{\mu \nu} & -3\left\{k_{1} \varphi_{\rho \sigma(\mu} \varphi_{\nu}\right)^{\rho \sigma}+k_{2} \varphi_{\mu \nu \rho} \varphi^{\rho}+k_{3} \varphi_{(\mu} \varphi_{\nu)} \\
& \left.-g_{\mu \nu}\left[\left(k_{1}+2 k_{4}\right) \varphi_{\rho \sigma \lambda} \varphi^{\rho \sigma \lambda}+\left(k_{2}+k_{3}+2 k_{5}\right) \varphi_{\lambda} \varphi^{\lambda}\right]\right\} .
\end{aligned}
$$

In conclusion, in three dimensions there exists a two-derivative coupling between gravity and a spin-3 field, that at $\mathcal{O}\left(\varphi^{2}\right)$ is unique up to field redefinitions. On the other hand, it is not clear a priori what is the counterpart of the frame-like action (1.2) under the map (1.3). This issue will now be addressed. It will lead to definite values for the $k_{i}$.

\footnotetext{
${ }^{6} \mathrm{On}(A) d S_{3}$ backgrounds all contributions of the form $R \ldots \varphi \ldots \varphi$.. become mass-like terms proportional to $\ell^{-2}$ as the ones that we already included in (2.8). Therefore not all parameters in (2.8) can be free: on $(A) d S_{3}$ one has to recover the Fronsdal "mass" [41], whose gauge variation cancels (2.5) on constantcurvature backgrounds. This is guaranteed by $(2.10)$.
} 


\subsection{Relation with the Chern-Simons action}

It is rather straightforward to construct solutions of the theory in its CS formulation: the extrema of the action are flat connections $e$ and $\omega$, with the condition that the gravity dreibein is invertible. The map (1.3) then allows to construct the metric and the spin-3 field explicitly. For a class of asymptotically AdS solutions this was done in [19] leading to

$$
\begin{aligned}
g & =\ell^{2} \frac{d r^{2}}{r^{2}}-\left\{r^{2}+(8 \pi G \ell)^{2}\left(\frac{\mathcal{L}\left(x^{+}\right) \widetilde{\mathcal{L}}\left(x^{-}\right)}{r^{2}}-\frac{\ell^{2}}{4} \frac{\mathcal{W}\left(x^{+}\right) \widetilde{\mathcal{W}}\left(x^{-}\right)}{r^{4}}\right)\right\} d x^{+} d x^{-} \\
& -8 \pi G \ell\left(\mathcal{L}\left(x^{+}\right)\left(d x^{+}\right)^{2}+\widetilde{\mathcal{L}}\left(x^{-}\right)\left(d x^{-}\right)^{2}\right) \\
\varphi & =-\frac{\ell}{8}(8 \pi G \ell)\left\{\left(\mathcal{W}\left(x^{+}\right)\left(d x^{+}\right)^{3}+\widetilde{\mathcal{W}}\left(x^{-}\right)\left(d x^{-}\right)^{3}\right)\right. \\
& +(8 \pi G \ell)\left(2 \frac{\widetilde{\mathcal{L}}\left(x^{-}\right) \mathcal{W}\left(x^{+}\right)}{r^{2}}+(8 \pi G \ell) \frac{\mathcal{L}\left(x^{+}\right)^{2} \widetilde{\mathcal{W}}\left(x^{-}\right)}{r^{4}}\right)\left(d x^{+}\right)^{2} d x^{-} \\
& \left.+(8 \pi G \ell)\left(2 \frac{\mathcal{L}\left(x^{+}\right) \widetilde{\mathcal{W}}\left(x^{-}\right)}{r^{2}}+(8 \pi G \ell) \frac{\widetilde{\mathcal{L}}\left(x^{-}\right)^{2} \mathcal{W}\left(x^{+}\right)}{r^{4}}\right)\left(d x^{-}\right)^{2} d x^{+}\right\}
\end{aligned}
$$

Here $r$ denotes a radial coordinate and $x^{ \pm}=\frac{t}{\ell} \pm \theta$.

We will now fix the coefficients $k_{i}$ by requiring that these fields also solve the equations of motion derived from the action (2.6), to the lowest non-trivial order in $\varphi$. This will lead to (1.5). The equation of motion for the metric is

$$
R_{\mu \nu}-\frac{1}{2} g_{\mu \nu} R-\frac{1}{\ell^{2}} g_{\mu \nu}=-\frac{1}{\sqrt{-g}} \frac{\delta\left(\sqrt{-g} \mathcal{L}_{F}\right)}{\delta g^{\mu \nu}} .
$$

The r.h.s. is the energy-momentum tensor of the spin-3 field, whose explicit expression is somewhat lengthy and will not be displayed. The equation of motion for $\varphi$ is

$$
\begin{aligned}
& \mathcal{F}_{\mu \nu \rho}-\frac{3}{2} g_{(\mu \nu} \mathcal{F}_{\rho)}+\left(\frac{m_{1}}{\ell^{2}}+3 k_{4} R\right) \varphi_{\mu \nu \rho}+\left(\frac{m_{2}}{\ell^{2}}+3 k_{5} R\right) g_{(\mu \nu} \varphi_{\rho)} \\
& +3 k_{1} R_{\lambda(\mu} \varphi_{\nu \rho)}{ }^{\lambda}+\frac{3}{2} k_{2} R_{(\mu \nu} \varphi_{\rho)}+\frac{3}{2} k_{2} g_{(\mu \nu} \varphi_{\rho)}^{\lambda \sigma} R_{\lambda \sigma}+3 k_{3} g_{(\mu \nu} R_{\rho) \sigma} \varphi^{\sigma}=0 .
\end{aligned}
$$

In this way we find a unique solution for the coefficients $k_{i}$ :

$$
k_{1}=\frac{3}{2}, \quad k_{2}=0, \quad k_{3}=-\frac{3}{4}, \quad k_{4}=-\frac{1}{2}, \quad k_{5}=0 .
$$

We remark that substituting (2.16) in the gauge transformation of the metric (2.11), all terms where the derivative acts on the parameter $\xi^{\rho \sigma}$ vanish. Moreover, the fields (2.13) 
solve the equations of motion (2.14) and (2.15) only at the lowest-order in $\mathcal{W}$ and $\widetilde{\mathcal{W}}$. Therefore, one has to add $\mathcal{O}\left(\varphi^{4}\right)$ corrections to the Lagrangian (2.8).

The solutions (2.13) are thus rich enough to fix all coefficients $k_{i}$, even if they do not parameterise the whole space of solutions of (1.2). For instance, they do not include black holes with higher-spin charges, as constructed in $[32,33,35,36]$, but we checked explicitly that these also solve the metric-like equations of motion. In sec. 3 we confirm the values (2.16) for the coefficients by a direct elimination of the auxiliary fields from the frame-like action, which guarantees that all fields constructed via the map (1.3) from solutions of the frame-like theory solve the equations of motion (2.14) and (2.15). This argument does not depend on the presence of a cosmological constant, and the coefficients (2.16) thus identify the metric-like counterpart of the action (1.2) also in Minkowski or de Sitter backgrounds.

\subsection{Algebra of gauge transformations}

We will now present the algebra of metric-like gauge transformations, up to the accessible orders in $\varphi$, and show that it closes on shell. Recall that the algebra generated by the frame-like transformations (1.4) closes off shell, but it still contains the auxiliary fields which are eliminated, via their equations of motion, in the metric formulation (see sec. 3). To compute the algebra one has to specify the tensorial nature of fields and gauge parameters. In analogy with gravity, we assume that gauge fields are symmetric covariant tensors, while gauge parameters are symmetric contravariant tensors. Moreover, we impose the trace constraint on the gauge parameter with a projector built from the metric.

The additional symmetry of the diffeomorphism invariant action (2.6) (with coefficients fixed as in (2.16) for simplicity) can thus be cast in the form ${ }^{7}$

$$
\begin{aligned}
\delta_{\xi} \varphi_{\mu \nu \rho} & =3\left(g_{\lambda(\mu} g_{\nu \mid \sigma}-\frac{1}{3} g_{\lambda \sigma} g_{(\mu \nu \mid}\right) \nabla_{\mid \rho)} \xi^{\lambda \sigma}+\mathcal{O}\left(\varphi^{2}\right) \\
\delta_{\xi} g_{\mu \nu} & =12 \xi^{\rho \sigma}\left\{\nabla_{\rho} \varphi_{\mu \nu \sigma}-2 \nabla_{(\mu} \varphi_{\nu) \rho \sigma}-g_{\rho \sigma} g^{\lambda \tau}\left[\nabla_{\lambda} \varphi_{\mu \nu \tau}-2 \nabla_{(\mu} \varphi_{\nu) \lambda \tau}\right]\right. \\
& +2 g_{\rho(\mu \mid} g^{\lambda \tau}\left[\nabla_{\lambda} \varphi_{\mid \nu) \sigma \tau}-\nabla_{\sigma} \varphi_{\mid \nu) \lambda \tau}-\nabla_{\mid \nu)} \varphi_{\sigma \lambda \tau}\right]-g_{\mu \nu} g^{\lambda \tau}\left[\nabla_{\lambda} \varphi_{\rho \sigma \tau}-2 \nabla_{\rho} \varphi_{\sigma \lambda \tau}\right] \\
& \left.+\frac{1}{2}\left(g_{\mu \rho} g_{\nu \sigma}-g_{\mu \nu} g_{\rho \sigma}\right) g^{\lambda \gamma} g^{\alpha \beta} \nabla_{\lambda} \varphi_{\alpha \beta \gamma}\right\}+\mathcal{O}\left(\varphi^{3}\right),
\end{aligned}
$$

where now $\xi^{\rho \sigma}$ is a traceful tensor. The extra terms appearing in (2.17) compared to (1.7) are those needed to implement the traceless projection, i.e. they are introduced by the

\footnotetext{
${ }^{7} v, w$ are the vector fields which generate diffeomorphisms and $\xi, \kappa$ the tensor fields which generate spin-3 transformations.
} 
substitution

$$
\xi^{\mu \nu} \rightarrow\left(\delta_{\rho}^{\mu} \delta_{\sigma}^{\nu}-\frac{1}{3} g_{\rho \sigma} g^{\mu \nu}\right) \xi^{\rho \sigma} .
$$

The issue is that the trace condition involves the metric which itself transforms under a second transformation on $\delta_{\xi} \varphi$.

Diffeomorphisms generate an off-shell subalgebra since they satisfy

$$
\left[\delta_{v}, \delta_{w}\right] g_{\mu \nu}=\delta_{[v, w]} g_{\mu \nu}, \quad\left[\delta_{v}, \delta_{w}\right] \varphi_{\mu \nu \rho}=\delta_{[v, w]} \varphi_{\mu \nu \rho}
$$

where

$$
[v, w]^{\mu}=v^{\nu} \partial_{\nu} w^{\mu}-w^{\nu} \partial_{\nu} v^{\mu}
$$

is the Lie bracket of the two vector fields $v$ and $w$. The commutator of a diffeomorphism with a spin-3 transformation can be cast in a similar form. For instance,

$$
\left[\delta_{v}, \delta_{\xi}\right] \varphi_{\mu \nu \rho}=3\left(g_{\lambda(\mu} g_{\nu \mid \sigma}-\frac{1}{3} g_{\lambda \sigma} g_{(\mu \nu \mid}\right) \nabla_{\mid \rho)}[v, \xi]^{\lambda \sigma}
$$

The resulting spin-3 transformation is generated by the Lie derivative of $\xi^{\mu \nu}$ along $v^{\sigma}$,

$$
[v, \xi]^{\mu \nu}=v^{\sigma} \partial_{\sigma} \xi^{\mu \nu}-2 \xi^{\sigma(\mu \mid} \partial_{\sigma} v^{\mid \nu)}
$$

A similar result holds for the metric, so that

$$
\left[\delta_{v}, \delta_{\xi}\right] g_{\mu \nu}=\delta_{[v, \xi]} g_{\mu \nu}, \quad\left[\delta_{v}, \delta_{\xi}\right] \varphi_{\mu \nu \rho}=\delta_{[v, \xi]} \varphi_{\mu \nu \rho}
$$

Note that $[v, \xi]^{\mu \nu}$ is not traceless even for a traceless $\xi^{\mu \nu}$, but this is not a problem thanks to the projector which multiplies it. Moreover, (2.23) remains true in arbitrary space-time dimensions. The peculiarities of the three-dimensional case thus manifest themselves in the commutator of two spin-3 transformations.

We were able to evaluate the commutators (2.19) and (2.23) because a diffeomorphism generates terms that are linear in the field on which it acts. Therefore, different orders in the expansion in powers of $\varphi$ cannot mix, and we expect that (2.23) continue to hold order by order in $\varphi$. The situation is very different when one considers commutators of two spin-3 transformations: these are not linear in the fields and, as a result, different orders could mix in the commutator. When the commutator acts on the spin-3 field, the higher-order corrections to (2.17) could even have an effect at lowest order. In fact, the gauge variation of $\varphi$ is schematically of the form

$$
\delta \varphi \sim g^{2} \nabla \xi+\mathcal{O}\left(\varphi^{2}\right)
$$

In a second gauge transformation the $\mathcal{O}(\varphi)$ variation of the metric mixes with the corresponding terms coming from the variation of the (yet unknown) $\mathcal{O}\left(\varphi^{2}\right)$ corrections. The only conclusion that we can draw from this commutator is that we do need corrections, since in their absence it is not possible to obtain a diffeomorphism out of $\left[\delta_{\kappa}, \delta_{\xi}\right] \varphi_{\mu \nu \rho}$. 
On the contrary, when the commutator acts on the metric there is a term of order zero in $\varphi$ that is insensitive to any correction to (2.17) and reads

$$
\begin{aligned}
& {\left[\delta_{\kappa}, \delta_{\xi}\right] g_{\mu \nu}=12 \xi^{\rho \sigma}\left\{\nabla_{\rho} \nabla_{\sigma} \kappa_{\mu \nu}+2 \nabla_{\rho} \nabla_{(\mu} \kappa_{\nu) \sigma}-4 \nabla_{(\mu \mid} \nabla_{\rho} \kappa_{\mid \nu) \sigma}-2 \nabla_{(\mu} \nabla_{\nu)} \kappa_{\rho \sigma}\right.} \\
& +2 g_{\rho(\mu \mid}\left[\square \kappa_{\mid \nu) \sigma}+\nabla_{\lambda} \nabla_{\mid \nu)} \kappa_{\sigma}{ }^{\lambda}+\nabla_{\lambda} \nabla_{\sigma} \kappa_{\mid \nu)}{ }^{\lambda}-2 \nabla_{\mid \nu)} \nabla_{\lambda}{\kappa_{\sigma}}^{\lambda}-2 \nabla_{\sigma} \nabla_{\lambda} \kappa_{\mid \nu)}{ }^{\lambda}\right] \\
& \left.-g_{\mu \nu}\left[\square \kappa_{\rho \sigma}+2 \nabla_{\lambda} \nabla_{\rho} \kappa_{\sigma}{ }^{\lambda}-4 \nabla_{\rho} \nabla_{\lambda} \kappa_{\sigma}{ }^{\lambda}\right]+g_{\mu \rho} g_{\nu \sigma} \nabla_{\lambda} \nabla_{\tau} \kappa^{\lambda \tau}\right\} \\
& -(\xi \leftrightarrow \kappa)+\mathcal{O}\left(\varphi^{2}\right) .
\end{aligned}
$$

For simplicity we presented the result in terms of traceless parameters; inserting (2.18) one recovers the full expression. At this order the right-hand side cannot contain a spin-3 transformation (that is at least linear in $\varphi$ ), so that it must be a diffeomorphism in order to grant the closure of the algebra. This can be made manifest rewriting (2.25) as

$$
\begin{aligned}
& {\left[\delta_{\kappa}, \delta_{\xi}\right] g_{\mu \nu}=-36\left\{\nabla_{(\mu \mid}\left(\xi^{\rho \sigma} \nabla_{\mid \nu)} \kappa_{\rho \sigma}-\kappa^{\rho \sigma} \nabla_{\mid \nu)} \xi_{\rho \sigma}\right)\right.} \\
& -3\left(\xi^{\rho \sigma} R_{\rho \sigma} \kappa_{\mu \nu}-\kappa^{\rho \sigma} R_{\rho \sigma} \xi_{\mu \nu}\right)+4\left(\xi^{\rho \sigma} R_{\rho(\mu} \kappa_{\nu) \sigma}-\kappa^{\rho \sigma} R_{\rho(\mu} \xi_{\nu) \sigma}\right) \\
& \left.-\xi^{\rho \sigma} Y_{\{2,2\}}^{T} \nabla_{(\rho} \nabla_{\sigma)} \kappa_{\mu \nu}+\kappa^{\rho \sigma} Y_{\{2,2\}}^{T} \nabla_{(\rho} \nabla_{\sigma)} \xi_{\mu \nu}\right\}+\mathcal{O}\left(\varphi^{2}\right),
\end{aligned}
$$

where $Y_{\{2,2\}}^{T}$ denotes the projector onto the traceless $\{2,2\}$ irrep of the group of permutations acting on the space-time indices $\mu, \nu, \rho, \sigma$. It can be built in terms of

$$
\begin{aligned}
\mathcal{T}_{\mu \nu, \rho \sigma} \equiv Y_{\{2,2\}} \nabla_{(\mu} \nabla_{\nu)} \xi_{\rho \sigma}=\frac{1}{6} & \left(2 \nabla_{(\mu} \nabla_{\nu)} \xi_{\rho \sigma}-\nabla_{\rho} \nabla_{(\mu} \xi_{\nu) \sigma}-\nabla_{\sigma} \nabla_{(\mu} \xi_{\nu) \rho}\right. \\
& \left.-\nabla_{\mu} \nabla_{(\rho} \xi_{\sigma) \nu}-\nabla_{\nu} \nabla_{(\rho} \xi_{\sigma) \mu}+2 \nabla_{(\rho} \nabla_{\sigma)} \xi_{\mu \nu}\right)
\end{aligned}
$$

as

$$
\begin{aligned}
Y_{\{2,2\}}^{T} \nabla_{(\mu} \nabla_{\nu)} \xi_{\rho \sigma}=\mathcal{T}_{\mu \nu, \rho \sigma} & -\left(g_{\mu \nu} \mathcal{T}_{\rho \sigma}-g_{\rho(\mu} \mathcal{T}_{\nu) \sigma}-g_{\sigma(\mu} \mathcal{T}_{\nu) \rho}+g_{\rho \sigma} \mathcal{T}_{\mu \nu}\right) \\
+ & \frac{1}{2}\left(g_{\mu \nu} g_{\rho \sigma}-g_{\rho(\mu} g_{\nu) \sigma}\right) \mathcal{T}
\end{aligned}
$$

where we defined $\mathcal{T}_{\mu \nu} \equiv \mathcal{T}_{\mu \nu, \lambda}{ }^{\lambda}$ and $\mathcal{T} \equiv \mathcal{T}_{\rho}{ }^{\rho}{ }_{, \sigma}{ }^{\sigma}$. The resulting combination has the same symmetries as the Weyl tensor and thus vanishes in three dimensions. In conclusion, we recovered a diffeomorphism generated $b y^{8}$

$$
v^{\mu}=18 g^{\mu \nu}\left\{\kappa^{\rho \sigma} \nabla_{\nu} \xi_{\rho \sigma}-\xi^{\rho \sigma} \nabla_{\nu} \kappa_{\rho \sigma}-\frac{1}{3}\left(\kappa_{\rho}^{\rho} \nabla_{\nu} \xi_{\sigma}^{\sigma}-\xi_{\rho}^{\rho} \nabla_{\nu} \kappa_{\sigma}^{\sigma}\right)\right\},
$$

plus a remainder proportional to the Ricci tensor. However, one can rewrite it in terms of the Einstein tensor: if one performs the substitution

$$
R_{\mu \nu} \rightarrow R_{\mu \nu}-\frac{1}{2} g_{\mu \nu}\left(R+\frac{2}{\ell^{2}}\right)
$$

${ }^{8}$ With respect to $(2.25)$ and $(2.26)$ we reinserted here the full dependence on the metric, so that $\kappa^{\alpha \beta}$ and $\xi^{\alpha \beta}$ are traceful tensors. 
the contributions in $g_{\mu \nu}$ cancel either identically or on account of the tracelessness of the parameters. At this order in $\varphi$ the field equations for the metric are the Einstein equations, so that the remainder in (2.26) signals that the algebra of gauge transformations closes only on shell.

\section{Mapping frame- to metric-like formulation}

In the last section we identified the metric-like theory that corresponds to the higher-spin theory defined by the frame-like action (1.2) to the first non-trivial order by analysing its equations of motion. Alternatively, one can identify it directly by eliminating the spin connections. This will be the content of this section. This also leads to an identification between the parameters of frame- and metric-like gauge symmetries.

\subsection{Elimination of spin connections}

To go from the frame-like formulation to the metric-like formulation, we have to solve the torsion constraint (the equation of motion for $\omega$ derived from the action (1.2)),

$$
d e+\omega \wedge e+e \wedge \omega=0
$$

to express the generalised spin connection $\omega$ in terms of the generalised vielbein $e$. In components this constraint reads

$$
\partial_{[\mu} e_{\nu]}^{\mathcal{C}}+f_{\mathcal{A B}}^{\mathcal{C}} \omega_{[\mu}^{\mathcal{A}} e_{\nu]}^{\mathcal{B}}=0 \quad \Leftrightarrow \quad \mathcal{D}_{[\mu} e_{\nu]}^{\mathcal{C}}=0
$$

where the curly letters are labels for the generators $\left\{J_{\mathcal{A}}\right\}$ of $\operatorname{sl}(3, \mathbb{R})$, and $\mathcal{D}_{\mu}$ denotes the full $s l(3)$ covariant connection including Levi-Civita and $s l(3)$ spin connection. The torsion constraint (3.2) together with the metric being covariantly constant,

$$
\nabla_{\mu} g_{\nu \rho}=\mathcal{D}_{\mu} g_{\nu \rho}=0
$$

lead to

$$
\kappa_{\mathcal{A B}} e_{\rho}^{\mathcal{A}} \mathcal{D}_{\mu} e_{\nu}^{\mathcal{B}}=0
$$

For $\mathfrak{g}=\operatorname{sl}(2, \mathbb{R})$ one could multiply this expression by the inverse vielbein to conclude that the vielbein is covariantly constant under the full connection (vielbein postulate), which allows to solve for the spin connection. For general Lie algebras this is not directly possible, and instead we will solve for the spin connection in a perturbative expansion. For that we separate the generators into the $s l(2)$ generators $\left\{J_{a}\right\}$ (labelled by small Latin indices), and the remaining generators $\left\{J_{A}\right\}$ (labelled by capital Latin indices and chosen to be orthogonal to the $J_{a}$ with respect to the Killing form). The relation (3.4) can be separated, and one obtains

$$
\mathcal{D}_{\mu} e_{\nu}^{c}=-\kappa_{A B} \stackrel{(0)}{g} \rho \lambda e_{\lambda}^{c} e_{\rho}^{A} \mathcal{D}_{\mu} e_{\nu}^{B},
$$


where we used the inverse $\stackrel{(0)}{g}^{\mu \nu}$ of the $s l(2)$-part $\stackrel{(0)}{g}_{\mu \nu}$ of the metric,

$$
\stackrel{(0)}{g}_{\mu \nu} \equiv e_{\mu}^{a} e_{\nu}^{b} \kappa_{a b}
$$

Eq. (3.5) can be interpreted as the correction to the vielbein postulate of the $s l(2)$ components $e^{c}$ of the vielbein. It remains to also get an expression for the covariant derivative of the non-sl(2) components $e^{C}$, and for that we have to determine the spin connection. To this end we split the sum over $\mathcal{B}=(b, B)$ in $(3.2)$ and we arrive, after some simple manipulations, at

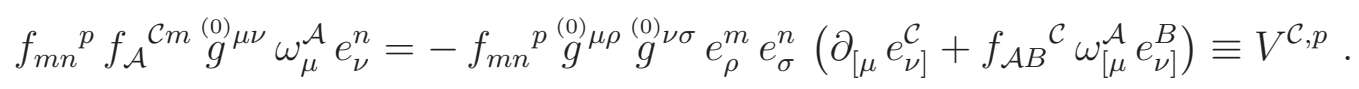

We will now solve (3.7) for $\omega$ in terms of $V$. By replacing the result for $\omega$ in the $\omega$ dependence of $V$ successively, we arrive at a perturbative expansion in the number of higher-spin vielbeins $e^{B}$.

We contract (3.7) with a suitable combination of structure constants, and arrive, after repeated use of the Jacobi identity and other identities collected in Appendix A, at

$$
\left(C_{2}\right)_{\mathcal{A}}^{\mathcal{D}} \omega_{\mu}^{\mathcal{A}} e_{\nu}^{b} \stackrel{(0)}{g}^{\mu \nu}=\left(\delta_{a}^{b} \delta_{\mathcal{C}}^{\mathcal{D}}+f_{a}^{b e} f_{e \mathcal{C}}{ }^{\mathcal{D}}+f_{a \mathcal{C}}{ }^{\mathcal{E}} f_{\mathcal{E}}{ }^{b \mathcal{D}}\right) V^{\mathcal{C}, a}
$$

Here, $C_{2}$ denotes the quadratic Casimir of $\operatorname{sl}(2)$ in the adjoint representation on $s l(3)$,

$$
\left(C_{2}\right)_{\mathcal{A}}^{\mathcal{D}}=f^{\mathcal{D}}{ }_{m \mathcal{C}} f^{\mathcal{C} m}{ }_{\mathcal{A}}
$$

The Casimir can of course be inverted as it is constant on every irreducible representation that occurs. In our convention the Casimir takes the value $s(s-1)$ if the corresponding $s l(2)$-representation has dimension $(2 s-1)$. Note that until now all manipulations were valid for general semi-simple Lie algebras. We now specialise to $\operatorname{sl}(3)$, and we find

$$
\begin{aligned}
& 2 \omega_{\beta}^{d}=e_{\beta}^{b}\left(\kappa_{a b} \delta_{c}^{d}+f_{a b}^{e} f_{e c}^{d}+f_{a c}^{e} f_{e b}^{d}\right) V^{c, a}, \\
& 6 \omega_{\beta}^{D}=e_{\beta}^{b}\left(\kappa_{a b} \delta_{C}^{D}+f_{a b}{ }^{e} f_{e C}{ }^{D}+f_{a C}{ }^{E} f_{E b}{ }^{D}\right) V^{C, a} .
\end{aligned}
$$

In the following, we will use the notation $e_{\mu}^{a}$ for $s l(2)$ components of the vielbein, and $E_{\mu}^{A}$ for the remaining components to better distinguish them. Similarly we use $\omega_{\mu}^{a}$ and $\Omega_{\mu}^{A}$. The lowest-order solutions for the spin connections then read

$$
\begin{aligned}
& \omega_{\beta}^{d}=-\frac{1}{2} e_{\beta}^{b}\left(f_{m n b} \delta_{c}^{d}+2 \kappa_{b[n} f_{m] c}{ }^{d}+2 \kappa_{c[n} f_{m] b}{ }^{d}\right) \stackrel{(0)}{g} \mu \rho \stackrel{(0)}{g} \nu \sigma e_{\rho}^{m} e_{\sigma}^{n} \partial_{[\mu} e_{\nu]}^{c}+\mathcal{O}\left(E^{2}\right), \\
& \Omega_{\beta}^{D}=-\frac{1}{6} e_{\beta}^{b} f_{m n}^{a}\left(\kappa_{a b} \delta_{C}^{D}+f_{a b}^{e} f_{e C}{ }^{D}+f_{a C}{ }^{E} f_{E b}{ }^{D}\right) \\
& \times \stackrel{(0)}{g} \mu \rho \stackrel{(0)}{g} \nu \sigma e_{\rho}^{m} e_{\sigma}^{n}\left(\partial_{[\mu} E_{\nu]}^{C}+f_{a B}^{C} \omega_{[\mu}^{a} E_{\nu]}^{B}\right) .
\end{aligned}
$$

Note that the second equation does not receive corrections of higher order in $E,{ }^{9}$ but replacing $\omega^{a}$ by its lowest order contribution will only yield $\Omega^{D}$ up to terms of order $\mathcal{O}\left(E^{3}\right)$

\footnotetext{
${ }^{9}$ There are no $\Omega^{A}$ appearing on the right-hand side due to the fact that in $\operatorname{sl}(3)$ the structure constants involving only non-sl(2) indices vanish, $f_{A B C}=0$.
} 
We could use these relations to rewrite the frame-like action (1.2) in terms of vielbeins, and then rewrite it in terms of metric $g$ and spin-3 field $\varphi$. Instead we will follow a different route by determining the gauge transformations of the metric-like theory from those in the frame-like theory.

\subsection{Relations between gauge parameters}

The frame-like theory is invariant under the gauge transformations (1.4), i.e. under generalised Lorentz transformations,

$$
\delta e_{\mu}^{\mathcal{C}}=f_{\mathcal{A B}}{ }^{\mathcal{C}} e_{\mu}^{\mathcal{A}} \Lambda^{\mathcal{B}}
$$

and generalised local translations,

$$
\delta e_{\mu}^{\mathcal{C}}=\partial_{\mu} \xi^{\mathcal{C}}+f_{\mathcal{A B}}{ }^{\mathcal{C}} \omega_{\mu}^{\mathcal{A}} \xi^{\mathcal{B}}
$$

In the frame-like description of pure gravity, diffeomorphisms are induced by local translations (see e.g. [42]). The same argument applies to the higher-spin setup: a local translation by

$$
\xi^{\mathcal{A}}=e_{\mu}^{\mathcal{A}} \xi^{\mu}
$$

can be decomposed as

$$
\delta e_{\mu}^{\mathcal{C}}=e_{\lambda}^{\mathcal{C}} \partial_{\mu} \xi^{\lambda}+\xi^{\lambda} \partial_{\lambda} e_{\mu}^{\mathcal{C}}+2 \xi^{\lambda}\left(\partial_{[\mu} e_{\lambda]}^{\mathcal{C}}+f_{\mathcal{A B}}{ }^{\mathcal{C}} \omega_{[\mu}^{\mathcal{A}} e_{\lambda]}^{\mathcal{B}}\right)+\xi^{\lambda} f_{\mathcal{A B}}{ }^{\mathcal{C}} \omega_{\lambda}^{\mathcal{A}} e_{\mu}^{\mathcal{B}} .
$$

The term in parentheses is proportional to the torsion constraint (3.2), so that up to a Lorentz-like transformation generated by $\Lambda^{\mathcal{B}}=-\xi^{\lambda} \omega_{\lambda}^{\mathcal{B}}$ the transformation is on-shell equivalent to a diffeomorphism generated by $-\xi^{\mu}$.

In the pure gravity case, one can invert this argument to conclude that any local translation by $\xi^{a}$ generates a diffeomorphism where the corresponding vector field is obtained by contracting $\xi^{a}$ with the inverse vielbein. In the higher-spin theory we expect that this expression is modified, and the simplest ansatz covariant in the $\operatorname{sl}(3)$ indices leads to

$$
\xi^{\mu}=g^{\mu \nu} \kappa_{\mathcal{A B}} e_{\nu}^{\mathcal{A}} \xi^{\mathcal{B}}
$$

This is consistent with the previous argument: if we insert $\xi^{\mathcal{A}}$ given in (3.15) into (3.17) we obtain an identity.

A general gauge parameter $\xi^{\mathcal{A}}$ will induce a spin-3 transformation as well as a diffeomorphism given by (3.17). On the other hand, we know from (3.15) that the diffeomorphism part is generated by

$$
\tilde{\xi}^{\mathcal{A}}=e_{\mu}^{\mathcal{A}} g^{\mu \nu} \kappa_{\mathcal{B C}} e_{\nu}^{\mathcal{C}} \xi^{\mathcal{B}} \equiv \mathcal{P}_{\mathcal{B}}^{\mathcal{A}} \xi^{\mathcal{B}}
$$

and we can identify $\mathcal{P}_{\mathcal{B}}^{\mathcal{A}}$ as the projector onto diffeomorphisms (that it is indeed a projector can be easily verified). This means in turn that the projector on pure spin-3 transformations is $(1-\mathcal{P})$. 
To find the correct ansatz for the spin-3 transformation we start from the linearised relation for a free spin-3 field,

$$
\xi^{a b}=3 \bar{e}_{\mu}^{a} \bar{e}_{\nu}^{b} \xi^{\mu \nu},
$$

where $\bar{e}$ denotes the vielbein of the fixed background (for which $\bar{e}_{\mu}^{A}=0$ ). We can rewrite this with the help of the symmetric structure constants $d_{\mathcal{A B C}}$ (see appendix A) as

$$
\xi^{A}=3 d_{a b}^{A} \bar{e}_{\mu}^{a} \bar{e}_{\nu}^{b} \xi^{\mu \nu}
$$

To obtain the corrections to this expression in the non-linear theory, we let the indices run over all $s l(3)$ labels and project the result by $(1-\mathcal{P})$ to ensure that no diffeomorphism is generated. This leads to the following ansatz for a pure spin-3 transformation generated by $\xi^{\mu \nu}$,

$$
\begin{aligned}
\xi^{\mathcal{A}} & =3\left(\delta_{\mathcal{B}}^{\mathcal{A}}-\mathcal{P}_{\mathcal{B}}^{\mathcal{A}}\right) \xi^{\mu \nu} e_{\mu}^{\mathcal{C}} e_{\nu}^{\mathcal{D}} d^{\mathcal{B}}{ }_{\mathcal{C D}} \\
& =3\left(\xi^{\mu \nu} e_{\mu}^{\mathcal{C}} e_{\nu}^{\mathcal{D}} d^{\mathcal{A}}{ }_{\mathcal{C D}}-d_{\mathcal{B C D}} e_{\rho}^{\mathcal{A}} e_{\sigma}^{\mathcal{B}} e_{\mu}^{\mathcal{C}} e_{\nu}^{\mathcal{D}} g^{\rho \sigma} \xi^{\mu \nu}\right)
\end{aligned}
$$

With this ansatz we can then derive the expression for the spin-3 transformation in the metric-like theory, which will be done in the following section. Note that this ansatz most likely needs to be modified at higher order in $E$, which becomes important if one computes higher order corrections to the gauge transformations.

\subsection{Transformations of metric-like fields}

Coming from the frame-like theory we can derive the spin-3 gauge transformations in the metric-like theory. The strategy is the following: we first express the metric and the spin-3 field in terms of vielbeins (see eq. (1.3)),

$$
g_{\mu \nu}=\kappa_{\mathcal{A B}} e_{\mu}^{\mathcal{A}} e_{\nu}^{\mathcal{B}}, \quad \varphi_{\mu \nu \rho}=\frac{1}{6} d_{\mathcal{A B C}} e_{\mu}^{\mathcal{A}} e_{\nu}^{\mathcal{B}} e_{\rho}^{\mathcal{C}}
$$

then use the gauge transformations (3.14) of the vielbeins under local translations by a parameter given in (3.21), insert the spin connection in terms of the vielbein (see eq. (3.12)), and finally express the result in terms of $g$ and $\varphi$.

Let us start with the transformation of the spin-3 field $\varphi$. Under a local translation it transforms as

$$
\delta \varphi_{\mu \nu \rho}=\frac{1}{2} d_{\mathcal{A B C}} e_{(\mu}^{\mathcal{A}} e_{\nu}^{\mathcal{B}} \mathcal{D}_{\rho)} \xi^{\mathcal{C}}
$$

Replacing the gauge parameter by the expression (3.21) and expanding in powers of $E$ we obtain

$$
\delta \varphi_{\mu \nu \rho}=\frac{3}{2} d_{A b c} d_{d e}^{A} e_{(\mu}^{b} e_{\nu}^{c} \mathcal{D}_{\rho)}\left(e_{\lambda}^{d} e_{\sigma}^{e} \xi^{\lambda \sigma}\right)+\mathcal{O}\left(E^{2}\right)
$$


The covariant derivative of the $\operatorname{sl}(2)$ components of the vielbein is of order $\mathcal{O}\left(E^{2}\right)$ (see eq. (3.5)), and we arrive at

$$
\begin{aligned}
\delta \varphi_{\mu \nu \rho} & =\frac{3}{2} d_{A b c} d_{d e}^{A} e_{\lambda}^{d} e_{\sigma}^{e} e_{(\mu}^{b} e_{\nu}^{c} \nabla_{\rho)} \xi^{\lambda \sigma}+\mathcal{O}\left(E^{2}\right) \\
& =3 \nabla_{(\mu}\left(\xi_{\nu \rho)}-\frac{1}{3} g_{\nu \rho)} g^{\lambda \sigma} \xi_{\lambda \sigma}\right)+\mathcal{O}\left(E^{2}\right),
\end{aligned}
$$

where we used the identity (A.9d) for the structure constants. This result equals (2.17a) including the projection of $\xi_{\lambda \sigma}$ to its traceless part.

We now consider the spin-3 transformation of the metric. Under a general local translation (3.14) it transforms as

$$
\delta g_{\mu \nu}=2 \kappa_{\mathcal{A B}} e_{(\mu}^{\mathcal{A}} \mathcal{D}_{\nu)} \xi^{\mathcal{B}}
$$

We replace the gauge parameter by the expression (3.21) for a pure spin-3 transformation, and after some manipulations where we also use (3.4) we arrive at

$$
\begin{aligned}
\delta g_{\mu \nu} & =-6 d_{\mathcal{A B C}} \xi^{\rho \sigma} e_{\rho}^{\mathcal{B}} e_{\sigma}^{\mathcal{C}} \mathcal{D}_{(\mu} e_{\nu)}^{\mathcal{A}} \\
& =-6 d_{A b c} \xi^{\rho \sigma} e_{\rho}^{b} e_{\sigma}^{c} \mathcal{D}_{(\mu} E_{\nu)}^{A}+\mathcal{O}\left(E^{3}\right)
\end{aligned}
$$

where in the last step we expanded in $s l(2)$ and non-sl(2) indices using that the covariant derivative of $e^{a}$ is of order $\mathcal{O}\left(E^{2}\right)$ (see eq. (3.5)). One observes that in (3.27) no derivatives of the gauge parameter $\xi^{\rho \sigma}$ appear. In fact one can show (see appendix B for the details) that this result precisely reproduces the gauge transformation (2.17b). Since there is a unique action at quadratic order in $\varphi$ that is invariant under this gauge transformation, we have again identified the action (1.5) as the metric-like counterpart of the frame-like action.

\section{$4 \quad$ Wald entropy for higher-spin black holes}

In ref. [32] the class of solutions considered in [19] was enlarged, in search for black holes with higher-spin charges; see also [33-36]. Although the presence of an event horizon is not a gauge-invariant statement in the theories we are considering (due to the Fronsdallike transformations (2.17)), there is a gauge where these solutions exhibit a regular event horizon [33]. This gauge is also supposed to be unique, and one can thus try to evaluate the entropy of higher-spin black holes using Wald's formula [37]. For a static black hole in three dimensions with metric

$$
g=g_{\rho \rho}(\rho) d \rho^{2}+g_{t t}(\rho) d t^{2}+g_{\theta \theta}(\rho) d \theta^{2}
$$

and regular horizon at $\rho=\rho_{h}$ it reads

$$
S=\left.\frac{\pi}{G} \sqrt{g_{\theta \theta}} g_{t t} g_{\rho \rho} \frac{\delta \mathcal{L}}{\delta R_{t \rho t \rho}}\right|_{\rho=\rho_{h}} .
$$


In all static solutions considered in [33-36] the spin-3 field takes the form

$$
\varphi=3 \varphi_{\rho \rho \theta}(\rho) d \rho^{2} d \theta+3 \varphi_{t t \theta}(\rho) d t^{2} d \theta+\varphi_{\theta \theta \theta}(\rho) d \theta^{3}
$$

Evaluating (4.2) taking into account (4.1) and (4.3) we find

$$
\begin{aligned}
S=\frac{\pi}{2 G} \sqrt{g_{\theta \theta}} & \left\{1+\frac{3}{2}\left[2\left(k_{4}+k_{5}\right)\left(g^{\theta \theta}\right)^{3}\left(\varphi_{\theta \theta \theta}\right)^{2}+2\left(k_{2}+2 k_{5}\right) g^{t t} g^{\rho \rho} g^{\theta \theta} \varphi_{t t \theta} \varphi_{\rho \rho \theta}\right.\right. \\
& +\left(2 k_{1}+k_{2}+6 k_{4}+2 k_{5}\right)\left(\left(g^{t t}\right)^{2} g^{\theta \theta}\left(\varphi_{t t \theta}\right)^{2}+\left(g^{\rho \rho}\right)^{2} g^{\theta \theta}\left(\varphi_{\rho \rho \theta}\right)^{2}\right) \\
& \left.\left.+\left(k_{2}+4 k_{5}\right)\left(g^{\theta \theta}\right)^{2}\left(g^{t t} \varphi_{t t \theta} \varphi_{\theta \theta \theta}+g^{\rho \rho} \varphi_{\rho \rho \theta} \varphi_{\theta \theta \theta}\right)\right]+\mathcal{O}\left(\varphi^{4}\right)\right\}\left.\right|_{\rho=\rho_{h}} .
\end{aligned}
$$

Substituting the values $(2.16)$ for the coefficients $k_{i}$ it simplifies to ${ }^{10}$

$$
S=\left.\frac{A}{4 G}\left\{1-\frac{3}{2}\left(g^{\theta \theta}\right)^{3}\left(\varphi_{\theta \theta \theta}\right)^{2}+\mathcal{O}\left(\varphi^{4}\right)\right\}\right|_{\rho=\rho_{h}}
$$

where $A=2 \pi \sqrt{g_{\theta \theta}\left(\rho_{h}\right)}$ is the length of the horizon. The same result can be recovered by taking advantage of the uniqueness of the two-derivative coupling up to field redefinitions. The field redefinition (2.12) cancels all terms with the Ricci tensor so that for the new action the black hole entropy is just one quarter of the area of the horizon, i.e.

$$
S=\frac{\pi}{2 G} \sqrt{g_{\theta \theta}^{(\text {new })}\left(\rho_{h}\right)}=\left.\frac{\pi}{2 G} \sqrt{g_{\theta \theta}}\left\{1+\frac{1}{2} g^{\theta \theta} \delta g_{\theta \theta}+\mathcal{O}\left(\varphi^{4}\right)\right\}\right|_{\rho=\rho_{h}} .
$$

Using (2.12) this can be shown to coincide with (4.4).

The black hole solutions of $[32,33,35,36]$ are constructed in such a way that they have a pointwise smooth BTZ limit if one switches off the spin-3 charge. We can then parameterise their mass, temperature and entropy by their deviation from the BTZ limits as

$$
\begin{aligned}
M & =M_{\mathrm{BTZ}}\left(1+\alpha_{M} \epsilon\right), \\
T & =T_{\mathrm{BTZ}}\left(1+\alpha_{T} \epsilon\right), \\
S & =S_{\mathrm{BTZ}}\left(1+\alpha_{S} \epsilon\right),
\end{aligned}
$$

where $\epsilon=0$ in the BTZ limit. In terms of the dimensionless parameters $\mathcal{L}$ and $\mathcal{W}$ used in [33], one has

$$
M_{\mathrm{BTZ}}=\frac{4 \pi}{\ell} \mathcal{L}, \quad T_{\mathrm{BTZ}}=\sqrt{\frac{2 \mathcal{L}}{\pi k \ell^{2}}}, \quad S_{\mathrm{BTZ}}=4 \pi \sqrt{2 \pi k \mathcal{L}},
$$

\footnotetext{
${ }^{10}$ The action (2.6) can be rewritten in many ways, e.g. by changing the order of covariant derivatives in the Fronsdal tensor which will shift some of the coefficients $k_{i}$. We have checked that the final expression for the entropy is unchanged.
} 
where $k=\frac{\ell}{4 G}$, and we set ${ }^{11}$

$$
\epsilon=\frac{\mathcal{W}^{2}}{\mathcal{L}^{3}}
$$

There are also higher-order corrections in $\epsilon$, but from our action we can only evaluate the deviation from the BTZ entropy (and the other thermodynamical parameters of the black hole) to the lowest non-trivial order in the spin-3 charge. Therefore, we restrict the discussion to $\mathcal{O}(\epsilon)$ terms. In addition there is also the spin-3 charge $Q$. It vanishes as $\mathcal{W} \rightarrow 0$ and we assume it to be of the form

$$
Q=\mathcal{W}\left(1+\alpha_{Q} \epsilon+\cdots\right)
$$

However, $\alpha_{Q}$ does not affect the following discussion, and to the order we are computing we can identify $Q$ with $\mathcal{W}$.

If one expresses the entropy as a function of $M$ and $Q$, the first law of black hole thermodynamics states

$$
\left(\frac{\partial S}{\partial M}\right)_{Q}=\frac{1}{T}
$$

We can use $(4.7 \mathrm{a})$ and $(4.8)$ to obtain $(\cdots$ are higher order terms in $\mathcal{W})$

$$
\mathcal{L}=\frac{\ell}{4 \pi} M-(4 \pi)^{2} \alpha_{M} \frac{\mathcal{W}^{2}}{\ell^{2} M^{2}}+\cdots
$$

Inserting this into the expression for the entropy we find

$$
S=2 \pi \sqrt{2 k M \ell}\left(1+32 \pi^{3}\left(2 \alpha_{S}-\alpha_{M}\right) \frac{\mathcal{W}^{2}}{\ell^{3} M^{3}}+\cdots\right),
$$

from where we compute

$$
\left(\frac{\partial S}{\partial M}\right)_{\mathcal{W}}=\pi \sqrt{\frac{2 k \ell}{M}}\left(1-160 \pi^{3}\left(2 \alpha_{S}-\alpha_{M}\right) \frac{\mathcal{W}^{2}}{\ell^{3} M^{3}}+\cdots\right) .
$$

On the other hand, for the temperature $(4.7 \mathrm{~b})$ we find

$$
\frac{1}{T}=\pi \sqrt{\frac{2 k \ell}{M}}\left(1-32 \pi^{3}\left(2 \alpha_{T}-\alpha_{M}\right) \frac{\mathcal{W}^{2}}{\ell^{3} M^{3}}+\cdots\right) .
$$

Comparison gives the linear relation for the coefficients

$$
5 \alpha_{S}-2 \alpha_{M}=\alpha_{T}
$$

Demanding regularity at the horizon,

$$
\frac{1}{T}=\left.2 \pi \sqrt{\frac{2 g_{\rho \rho}}{-g_{t t}^{\prime \prime}}}\right|_{\rho=\rho_{h}}=\left.2 \pi \sqrt{\frac{2 \varphi_{\rho \rho \theta}}{-\varphi_{t t \theta}^{\prime \prime}}}\right|_{\rho=\rho_{h}}
$$

\footnotetext{
${ }^{11}$ Our parameter $\epsilon$ is related to the parameter $\zeta$ used in [33] by $\epsilon=\frac{32 \pi}{k} \zeta^{2}$.
} 
the temperature is fixed in terms of $\mathcal{L}$ and $\mathcal{W}$, and for the solution presented in eqs. (4.13) and (C.1) of [33] this leads to

$$
\alpha_{T}=-\frac{15 k}{256 \pi}
$$

We can now evaluate Wald's formula (4.5) on the black hole solution of [33] to fix $\alpha_{S}$. The relation (4.16) would then fix also $\alpha_{M}$. To this end the overall normalisation of the fields is crucial, and we checked that the metric and the spin-3 field presented in [33] solve our equations of motion (2.14) and (2.15) provided that one multiplies their $\varphi_{\mu \nu \rho}$ by $1 / 6 .^{12}$ Eq. (4.5) eventually implies

$$
\alpha_{S}=\frac{9 k}{256 \pi}
$$

and together with (4.16) and (4.18)

$$
\alpha_{M}=\frac{15 k}{128 \pi}
$$

The correction to the BTZ entropy that we found in this way does not agree with the one presented in sec. 4.2 of [33] (see also [34,43] for a discussion of the proposal of [33] from a CFT perspective). On the other hand, the previous analysis suggests a possible interpretation of the mismatch: in [33] the entropy was derived from the first law (4.11) under the assumption $M=M_{\mathrm{BTZ}}$, i.e. $\alpha_{M}=0$. Inserting this ansatz in (4.16) one indeed reproduces the $\alpha_{S}$ of [33], while our result seems to suggest the need for $\mathcal{W}$ corrections to the mass. To test this possibility it would be desirable to compute the shift in the mass in an independent way. ${ }^{13}$ However, due to the modified asymptotic behaviour of the black hole solutions this is not straightforward: at infinity the metric has the same radial dependence as an AdS space with half the radius of the vacuum solution of our field equations (2.14) and (2.15). The discrete jump in the asymptotic behaviour could also create problems of convergence of the integrals involved in Wald's proof of the first law of black hole thermodynamics. This issue deserves further study.

\section{Discussion}

We considered the minimal coupling of Fronsdal's action to gravity in three space-time dimensions and we showed that at the lowest order in the higher-spin field it preserves the same amount of gauge symmetry as the free action. The resulting two-derivative coupling (that is not available in higher space-time dimensions [6-11]) is unique up to

\footnotetext{
${ }^{12}$ The need for this rescaling can also be inferred from the comparison between our definition for the metric-like fields (1.3) and the corresponding definition in [33].

${ }^{13} \mathrm{~A}$ modification of the relation $M=M_{\mathrm{BTZ}}$ was also proposed in [44] via a direct construction of the corresponding conserved charge in the CS formulation.
} 
field redefinitions, and it does not require a cosmological constant. In the spin-3 case we exhibited its relation with the $S L(3, \mathbb{R}) \times S L(3, \mathbb{R})$ Chern-Simons action that describes the coupling in a frame-like language. We also proved that a complete metric-like action would require higher-order corrections in the spin-3 field to preserve the gauge invariance, but the frame-like formulation indicates that neither fields of different spin nor higherderivative couplings are necessary.

A natural extension of the present work would be to identify the full metric-like counterpart of the $S L(3, \mathbb{R}) \times S L(3, \mathbb{R})$ Chern-Simons action. The major simplification of the spectrum in comparison to all known higher-spin gauge theories in $D \geq 4$ gives hope to achieve this goal, although the next order is already quite intricate and at present it is not even clear whether the action has to be polynomial in the spin-3 field. The situation is as if in gravity we only knew the action up to some order in the graviton field. Without an understanding of the geometric principles - covariant derivatives, curvatures, etc. and of the full nonlinear diffeomorphism symmetry, this action would look mysterious. In the higher-spin case the "geometric" structures which are implied by the extension of diffeomorphism to include higher-spin gauge symmetries are unknown. Some attempts in this direction are reported e.g. in [45-49], where reformulations of the free theory in terms of higher-spin curvatures were studied. In our setup progress in this direction might come from abandoning metric compatibility and starting from a Palatini-like description of the dynamics. The elimination of the auxiliary fields from the frame-like action, that we analysed in sec. 3, also gives hope that a simple way to group all non-linearities exists, but a detailed analysis of the next corrections is needed.

Another interesting direction to be explored is the extension of all previous considerations to $S L(N, \mathbb{R}) \times S L(N, \mathbb{R})$ Chern-Simons theories and to their $N \rightarrow \infty$ limits $[20,21]$, possibly including the matter couplings of [27] or generalisations thereof.

\section{Acknowledgements}

We performed or checked various computations with $x$ Act packages for Mathematica [50], and we are grateful to T. Nutma for his advise and for the use of his package xTras. A.C., S.F. and S.P. acknowledge the Erwin Schrödinger Institute in Vienna for hospitality, and the participants in the ESI workshop on Higher Spin Gravity for feedback on a presentation of some of the results contained in this paper. In particular, we would like to thank M. Ammon for a suggestion which provoked the analysis presented in sec. 4. We also thank M. Bañados, C. Eling, E. Joung, M. Henneaux, J. Mei, Y. Oz, M. Taronna and A. Virmani for discussions. 


\section{A Conventions}

A pair of parentheses denotes the symmetrisation of the indices it encloses with weight one, such that for instance

$$
A_{(\mu} B_{\nu)}=\frac{1}{2}\left(A_{\mu} B_{\nu}+A_{\nu} B_{\mu}\right)
$$

In a similar fashion a pair of square brackets denotes the antisymmetrisation of the indices it encloses. Note that this convention differs from the one employed in our earlier publication [19]. We adopt the mostly plus convention for the metric and our curvature conventions are

$$
\left[\nabla_{\mu}, \nabla_{\nu}\right] \omega_{\rho}=R_{\mu \nu \rho}^{\sigma} \omega_{\sigma}, \quad R_{\mu \nu}=R_{\mu \rho \nu}^{\rho} .
$$

We often omit contracted indices in the traces of a tensor: for instance $\varphi_{\mu} \equiv \varphi_{\mu \lambda}{ }^{\lambda}$.

The algebra $\operatorname{sl}(3, \mathbb{R})$ can be given in terms of generators $J_{a}$ and $T_{a b}$ with the commutation relations ${ }^{14}$

$$
\begin{aligned}
& {\left[J_{a}, J_{b}\right]=\epsilon_{a b c} J^{c},} \\
& {\left[J_{a}, T_{b c}\right]=2 \epsilon_{a(b}^{d} T_{c) d},} \\
& {\left[T_{a b}, T_{c d}\right]=-2\left(\eta_{a(c} \epsilon_{d) b e}+\eta_{b(c} \epsilon_{d) a e}\right) J^{e},}
\end{aligned}
$$

with $T_{[a b]}=\eta^{a b} T_{a b}=0$. The Levi-Civita symbol is defined such that

$$
\epsilon^{012}=-\epsilon_{012}=1,
$$

and indices are raised and lowered with $\eta_{a b}=\operatorname{diag}(-1,1,1)$. An explicit $3 \times 3$ matrix representation for the $T_{a b}$ is given by

$$
T_{a b}=\left(J_{a} J_{b}+J_{b} J_{a}-\frac{2}{3} \eta_{a b} J_{c} J^{c}\right),
$$

with $J_{a}$ in the three-dimensional representation of $\operatorname{sl}(2, \mathbb{R}) \hookrightarrow \operatorname{sl}(3, \mathbb{R})$. We denote by $\left\{J_{A}\right\}$ a set of five independent generators built from the $T_{a b}$, and the set of all generators $\left\{J_{a}, J_{A}\right\}$ is denoted by $\left\{J_{\mathcal{A}}\right\}$.

We normalise the Killing form to be one half of the matrix trace in the fundamental representation of $\operatorname{sl}(3, \mathbb{R})$,

$$
\kappa_{\mathcal{A B}}=\frac{1}{2} \operatorname{tr}\left(J_{\mathcal{A}} J_{\mathcal{B}}\right)
$$

such that e.g. $\kappa_{a b}=\eta_{a b}$ and $\kappa_{a B}=0$. The anti-symmetric and symmetric structure constants are defined as

$$
\begin{aligned}
f_{\mathcal{A B C}} & =\frac{1}{2} \operatorname{tr}\left(\left[J_{\mathcal{A}}, J_{\mathcal{B}}\right] J_{\mathcal{C}}\right), \\
d_{\mathcal{A B C}} & =\frac{1}{2} \operatorname{tr}\left(\left\{J_{\mathcal{A}}, J_{\mathcal{B}}\right\} J_{\mathcal{C}}\right),
\end{aligned}
$$

\footnotetext{
${ }^{14}$ With respect to $[19]$ we choose $\sigma=-1$.
} 
such that $f_{a b c}=\epsilon_{a b c}$ and $d_{a b c}=0$. The structure constants satisfy a number of identities, and in the main text we use

$$
\begin{aligned}
f_{a}^{b c} f_{b c}^{d} & =-2 \delta_{a}^{d} \\
f_{a b}^{c} f_{c}^{d e} & =-\left(\delta_{a}^{d} \delta_{b}^{e}-\delta_{a}^{e} \delta_{b}^{d}\right) \\
d_{A b c} \kappa^{b c} & =0 \\
d_{A b c} d_{d e}^{A} & =-\frac{2}{3} \kappa_{b c} \kappa_{d e}+2 \kappa_{d(b} \kappa_{c) e}
\end{aligned}
$$

\section{B Spin-3 transformation of the metric}

In this appendix we want to show that the spin-3 transformation of the metric in (3.27) derived from the frame-like formulation (which we call $\delta^{I} g_{\mu \nu}$ in the following) can be expressed in terms of metric $g$ and spin-3 field $\varphi$ as in $\left(2.17 \mathrm{~b}\right.$ ) (which we will call $\delta^{I I} g_{\mu \nu}$ ).

We first expand the $\operatorname{sl}(3)$-covariant derivative $\mathcal{D}$ in (3.27) as the sum of the $\operatorname{sl}(2)$ covariant derivative $\stackrel{(0)}{\mathcal{D}}$ and the non-sl(2) part $\Omega$ of the spin connection,

$$
\delta^{I} g_{\mu \nu}=-6 d_{A b c} e_{\rho}^{b} e_{\sigma}^{c} \xi^{\rho \sigma}\left(\stackrel{(0)}{\mathcal{D}}_{(\mu} E_{\nu)}^{A}+f_{B d}^{A} \Omega_{(\mu}^{B} e_{\nu)}^{d}\right)+\mathcal{O}\left(E^{3}\right)
$$

and replace $\Omega$ by the expression $(3.12 \mathrm{~b})$ to arrive at

$$
\begin{aligned}
\delta^{I} g_{\mu \nu} & =-\xi^{\rho \sigma} e_{\rho}^{e} e_{\sigma}^{f} e_{(\mu}^{a} e_{\nu)}^{b} e_{\alpha}^{m} e_{\gamma}^{n} g^{\alpha \beta} g^{\gamma \delta} \stackrel{(0)}{\mathcal{D}}_{\beta} E_{\delta}^{A} \\
& \times\left(6 \kappa_{m(a} \kappa_{b) n} d_{A e f}+d^{D}{ }_{e f} f_{m n}{ }^{d} f_{C D(a}\left(\kappa_{b) d} \delta_{A}^{C}+f_{b) d}{ }^{g} f_{A g}{ }^{C}+f_{b) E}{ }^{C} f_{A d}{ }^{E}\right)\right)
\end{aligned}
$$

On the other hand we can start from the expression (2.17b) and expand it in vielbeins. For that we first augment the Levi-Civita covariant derivative $\nabla_{\mu}$ to the $s l(2)$-covariant

derivative $\stackrel{(0)}{\mathcal{D}} \mu$ because then it acts trivially on the $s l(2)$-components $e^{a}$ of the vielbein (up to $\mathcal{O}\left(E^{2}\right)$ corrections). We obtain

$$
\begin{aligned}
\delta^{I I} g_{\mu \nu}= & \xi^{\rho \sigma} e_{\rho}^{e} e_{\sigma}^{f} e_{(\mu}^{a} e_{\nu)}^{b} e_{\alpha}^{m} e_{\gamma}^{n} g^{\alpha \beta} g^{\gamma \delta} \stackrel{(0)}{\mathcal{D}}_{\beta} E_{\delta}^{A} \\
\times( & 4 \kappa_{n(a} d_{b) A(e} \kappa_{f) m}+2 d_{A a b} \kappa_{m(e} \kappa_{f) n}-4 d_{A e f} \kappa_{m(a} \kappa_{b) n}-8 \kappa_{m(a} d_{b) A(e} \kappa_{f) n} \\
& +4 d_{A m(e} \kappa_{f)(a} \kappa_{b) n}+4 d_{A m(a} \kappa_{b)(e} \kappa_{f) n}+4 d_{A(a \mid(e} \kappa_{f) \mid b)} \kappa_{m n}-8 d_{A n(a} \kappa_{b)(e} \kappa_{f) m} \\
& -8 d_{A n(e} \kappa_{f)(a} \kappa_{b) m}+2 d_{A m n} \kappa_{a(e} \kappa_{f) b}-2 d_{A e f} \kappa_{a b} \kappa_{m n}-4 d_{A m(e} \kappa_{f) n} \kappa_{a b} \\
& +8 d_{A n(e} \kappa_{f) m} \kappa_{a b}-2 d_{A m n} \kappa_{a b} \kappa_{e f}-2 d_{A a b} \kappa_{e f} \kappa_{m n}-4 d_{A m(a} \kappa_{b) n} \kappa_{e f} \\
& \left.+8 d_{A n(a} \kappa_{b) m} \kappa_{e f}\right) .
\end{aligned}
$$

Identifying the two expressions (B.2) and (B.3) then amounts to checking an identity for the structure constants which can easily be done with the help of a computer. 


\section{Tensors of arbitrary rank}

One can extend the considerations of sec. 2.1 to symmetric tensors of arbitrary rank. To this end it is convenient to switch to a more compact notation: in this section repeated covariant (or contravariant) indices denote a symmetrisation. Moreover, symmetrised indices belonging to the same tensor are substituted by a single Greek letter with a label counting the total number of indices. For instance, the covariantised Fronsdal tensor can be written as

$$
\mathcal{F}_{\mu_{s}}=\square \varphi_{\mu_{s}}-\frac{s}{2}\left(\nabla^{\lambda} \nabla_{\mu} \varphi_{\mu_{s-1} \lambda}+\nabla_{\mu} \nabla^{\lambda} \varphi_{\mu_{s-1} \lambda}\right)+\left(\begin{array}{l}
s \\
2
\end{array}\right) \nabla_{\mu} \nabla_{\mu} \varphi_{\mu_{s-2}},
$$

where $\varphi_{\mu_{s-2}}=g^{\rho \sigma} \varphi_{\mu_{s-2} \rho \sigma}$. With these conventions its variation under

$$
\delta \varphi_{\mu_{s}}=s \nabla_{\mu} \xi_{\mu_{s-1}}, \quad \text { with } \quad \xi_{\mu_{s-3}}=0
$$

takes essentially the same form as in the spin-3 case,

$$
\begin{aligned}
\delta \mathcal{F}_{\mu_{s}}= & -6\left(\begin{array}{l}
s \\
3
\end{array}\right) \xi^{\alpha \beta}{ }_{\mu_{s-3}} \nabla_{\mu} R_{\alpha \mu \mu \beta}-9\left(\begin{array}{l}
s \\
3
\end{array}\right) R_{\alpha \mu \mu \beta} \nabla_{\mu} \xi_{\mu_{s-3}}{ }^{\alpha \beta}+2\left(\begin{array}{l}
s \\
2
\end{array}\right) R_{\alpha \mu \mu \beta} \nabla^{\alpha} \xi_{\mu_{s-2} \beta} \\
& -2\left(\begin{array}{l}
s \\
2
\end{array}\right) \xi^{\alpha}{ }_{\mu_{s-2}} \nabla_{\alpha} R_{\mu \mu}+\frac{s}{2} R_{\mu \alpha} \nabla^{\alpha} \xi_{\mu_{s-1}}-3\left(\begin{array}{l}
s \\
2
\end{array}\right) R_{\mu \alpha} \nabla_{\mu} \xi_{\mu_{s-2}}{ }^{\alpha}
\end{aligned}
$$

In Fronsdal's approach the double trace of the fields is forced to vanish. As a result, at the lowest order in $\varphi$ the spin-3 example already captures all features of the general case because one cannot construct other curvature terms than those in (2.8). The most general Lagrangian that is quadratic in $\varphi$ and contains at most two derivatives is

$$
\begin{aligned}
\mathcal{L}_{F} & =\frac{\sqrt{-g}}{16 \pi G}\left\{\varphi^{\mu_{s}}\left(\mathcal{F}_{\mu_{s}}-\frac{1}{2}\left(\begin{array}{l}
s \\
2
\end{array}\right) g_{\mu \mu} \mathcal{F}_{\mu_{s-2}}\right)+\frac{1}{\ell^{2}}\left(m_{1} \varphi_{\mu_{s}} \varphi^{\mu_{s}}+m_{2} \varphi_{\mu_{s-2}} \varphi^{\mu_{s-2}}\right)\right. \\
& +\left(\begin{array}{l}
s \\
2
\end{array}\right) R_{\alpha \beta}\left(k_{1} \varphi^{\alpha}{ }_{\mu_{s-1}} \varphi^{\beta \mu_{s-1}}+k_{2} \varphi^{\alpha \beta}{ }_{\mu_{s-2}} \varphi^{\mu_{s-2}}+(s-2) k_{3} \varphi^{\alpha}{ }_{\mu_{s-3}} \varphi^{\beta \mu_{s-3}}\right) \\
& \left.+\left(\begin{array}{l}
s \\
2
\end{array}\right) R\left(k_{4} \varphi_{\mu_{s}} \varphi^{\mu_{s}}+k_{5} \varphi_{\mu_{s-2}} \varphi^{\mu_{s-2}}\right)\right\} .
\end{aligned}
$$

Requiring that on $(\mathrm{A}) \mathrm{dS}$ the terms in $\ell^{-2}$ build the Fronsdal mass term [41] then implies

$$
\begin{aligned}
& m_{1}=s\left\{(s-1)\left(k_{1}+3 k_{4}\right)-\frac{3 s-5}{2}\right\} \\
& m_{2}=s(s-1)\left\{k_{2}+(s-2) k_{3}+3 k_{5}+\frac{s(3 s-1)-6}{8}\right\} .
\end{aligned}
$$


If these conditions are satisfied, the Lagrangian $\mathcal{L}=\mathcal{L}_{E H}+\mathcal{L}_{F}$ is invariant, up to linear order in $\varphi$ and up to total derivatives, under the simultaneous transformations (C.2) and

$$
\begin{aligned}
\delta g_{\mu \mu} & =\left(\begin{array}{c}
s \\
2
\end{array}\right)\left\{a_{1} \varphi_{\mu \mu \alpha_{s-2}} \nabla \cdot \xi^{\alpha_{s-2}}+\left(a_{2}+b_{2}\right) \varphi_{\mu \alpha_{s-1}} \nabla_{\mu} \xi^{\alpha_{s-1}}+a_{3} \varphi_{\mu \alpha \beta_{s-2}} \nabla^{\alpha} \xi_{\mu} \beta_{s-2}\right. \\
& +a_{4} \varphi_{\mu \alpha_{s-3}} \nabla \cdot \xi_{\mu}^{\alpha_{s-3}}+\left(a_{5}+b_{5}\right) \varphi_{\alpha_{s-2}} \nabla_{\mu} \xi_{\mu}^{\alpha_{s-2}}+a_{6} \varphi_{\alpha \beta_{s-3}} \nabla^{\alpha} \xi_{\mu \mu}{ }^{\beta_{s-3}} \\
& +b_{1} \xi^{\alpha \beta_{s-2}} \nabla_{\alpha} \varphi_{\mu \mu \beta_{s-2}}+b_{2} \xi^{\alpha_{s-1}} \nabla_{\mu} \varphi_{\mu \alpha_{s-1}}+b_{3} \xi_{\mu}{ }^{\alpha_{s-2}} \nabla \cdot \varphi_{\mu \alpha_{s-2}} \\
& +b_{4} \xi_{\mu}{ }^{\alpha \beta_{s-3}} \nabla_{\alpha} \varphi_{\mu \beta_{s-3}}+b_{5} \xi_{\mu}^{\alpha_{s-2}} \nabla_{\mu} \varphi_{\alpha_{s-2}}+b_{6} \xi_{\mu \mu}^{\alpha_{s-3}} \nabla \cdot \varphi_{\alpha_{s-3}} \\
& \left.+g_{\mu \mu}\left(c_{1} \varphi_{\alpha \beta_{s-1}} \nabla^{\alpha} \xi^{\beta_{s-1}}+c_{2} \varphi_{\alpha_{s-2}} \nabla \cdot \xi^{\alpha_{s-2}}+d_{1} \xi^{\alpha_{s-1}} \nabla \cdot \varphi_{\alpha_{s-1}}+d_{2} \xi^{\alpha \beta_{s-2}} \nabla_{\alpha} \varphi_{\beta_{s-2}}\right)\right\} .
\end{aligned}
$$

As in the spin-3 case, all coefficients in (C.7) are fixed except $b_{2}$ and $b_{5}$, which parameterise field-dependent diffeomorphisms.

The coefficients $a_{i}$ and $c_{i}$, which multiply terms with the derivative acting on the gauge parameter, depend on the $k_{i}$,

$$
\begin{array}{ll}
a_{1}=2 k_{2}, & a_{2}=\frac{4 s+2(s-1) k_{1}-2}{s-1}, \\
a_{3}=2(s-1)\left(k_{1}-2\right)+2, & a_{4}=(s-2)\left(2 s+4 k_{3}-3\right), \\
a_{5}=2\left(4 s+k_{2}-8\right), & a_{6}=(s-2) k_{2}, \\
c_{1}=-\frac{2 s(s-1)\left(k_{1}+2 k_{4}-2\right)+2 s^{2}}{s-1}, & \\
c_{2}=-4\left(k_{2}+(s-2) k_{3}+2 k_{5}\right)-s(s-2) . &
\end{array}
$$

The remaining coefficients do not depend on the free parameters in the Lagrangian:

$$
\begin{array}{lll}
b_{1}=4, & b_{3}=8(s-2), & b_{4}=-4(s-1)(s-2), \\
b_{6}=2(s-2), & d_{1}=-4(s-2), & d_{2}=2(s-2)(s+1) .
\end{array}
$$

As in the spin-3 case one can set to zero all terms with the derivative acting on $\xi$ by choosing $b_{2}=-a_{2}, b_{5}=-a_{5}$, and

$$
k_{1}=\frac{2 s-3}{s-1}, \quad k_{2}=0, \quad k_{3}=\frac{3-2 s}{4}, \quad k_{4}=-\frac{1}{2}, \quad k_{5}=\frac{(s-2)(s-3)}{8} .
$$

The coupling is again unique since one can remove all contributions in the Ricci tensor with the field redefinition

$$
\begin{aligned}
g_{\mu \mu}^{(\text {new })}=g_{\mu \mu} & -\left(\begin{array}{l}
s \\
2
\end{array}\right)\left\{k_{1} \varphi_{\mu \alpha_{s-1}} \varphi_{\mu}^{\alpha_{s-1}}+k_{2} \varphi_{\mu \mu \alpha_{s-2}} \varphi^{\alpha_{s-2}}+k_{3} \varphi_{\mu \alpha_{s-3}} \varphi_{\mu}^{\alpha_{s-3}}\right. \\
& \left.-g_{\mu \mu}\left[\left(k_{1}+2 k_{4}\right) \varphi_{\alpha_{s}} \varphi^{\alpha_{s}}+\left(k_{2}+k_{3}+2 k_{5}\right) \varphi_{\alpha_{s-2}} \varphi^{\alpha_{s-2}}\right]\right\} .
\end{aligned}
$$


Note that the terms in (C.4) should appear in the metric-like counterpart of any ChernSimons theory involving $\varphi_{\mu_{1} \cdots \mu_{s}}$ since they provide a diffeomorphism invariant version of Fronsdal's kinetic operator. On the other hand, we expect inequivalent higher-order completions of (C.4), corresponding to different Chern-Simons theories. The simplest examples of this type involve various higher-spin fields, and it is not clear whether for $s>4$ the gauge symmetry of (C.4) can also be preserved at higher orders without considering at the same time symmetric tensors of different rank (see also [51] for a direct construction of higher-spin interactions in the frame-like formulation).

\section{References}

[1] M. A. Vasiliev, "Consistent equation for interacting gauge fields of all spins in $(3+1)$ dimensions," Phys. Lett. B 243 (1990) 378, "Nonlinear equations for symmetric massless higher spin fields in (A)dS(d)," Phys. Lett. B 567 (2003) 139 [hep-th/0304049].

[2] X. Bekaert, S. Cnockaert, C. Iazeolla and M. A. Vasiliev, "Nonlinear higher spin theories in various dimensions," hep-th/0503128.

[3] A. Fotopoulos and M. Tsulaia, "Gauge Invariant Lagrangians for Free and Interacting Higher Spin Fields. A Review of the BRST formulation," Int. J. Mod. Phys. A 24 (2009) 1 [arXiv:0805.1346 [hep-th]].

[4] X. Bekaert, N. Boulanger and P. Sundell, "How higher-spin gravity surpasses the spin two barrier: no-go theorems versus yes-go examples," arXiv:1007.0435 [hep-th].

[5] A. Sagnotti, "Notes on Strings and Higher Spins," arXiv:1112.4285 [hep-th].

[6] A. K. H. Bengtsson, I. Bengtsson and L. Brink, "Cubic Interaction Terms For Arbitrary Spin," Nucl. Phys. B 227 (1983) 31.

[7] R. R. Metsaev, "Cubic interaction vertices of massive and massless higher spin fields," Nucl. Phys. B 759 (2006) 147 [hep-th/0512342].

[8] R. Manvelyan, K. Mkrtchyan and W. Rühl, "General trilinear interaction for arbitrary even higher spin gauge fields," Nucl. Phys. B 836 (2010) 204 [arXiv:1003.2877 [hep-th]].

[9] A. Sagnotti and M. Taronna, "String Lessons for Higher-Spin Interactions," Nucl. Phys. B 842 (2011) 299 [arXiv:1006.5242 [hep-th]].

[10] E. Joung, L. Lopez and M. Taronna, "Solving the Noether procedure for cubic interactions of higher spins in (A)dS," arXiv:1207.5520 [hep-th].

[11] M. A. Vasiliev, "Cubic Vertices for Symmetric Higher-Spin Gauge Fields in $(A) d S_{d}$," Nucl. Phys. B 862 (2012) 341 [arXiv:1108.5921 [hep-th]]. 
[12] X. Bekaert, N. Boulanger and S. Leclercq, "Strong obstruction of the Berends-Burgers-van Dam spin-3 vertex," J. Phys. A 43 (2010) 185401 [arXiv:1002.0289 [hep-th]].

[13] D. Polyakov, "Higher Spins and Open Strings: Quartic Interactions," Phys. Rev. D 83 (2011) 046005 [arXiv:1011.0353 [hep-th]].

[14] M. Taronna, "Higher-Spin Interactions: four-point functions and beyond," JHEP 1204 (2012) 029 [arXiv:1107.5843 [hep-th]].

[15] P. Dempster and M. Tsulaia, "On the Structure of Quartic Vertices for Massless Higher Spin Fields on Minkowski Background," arXiv:1203.5597 [hep-th].

[16] G. Barnich, M. Grigoriev, A. Semikhatov and I. Tipunin, "Parent field theory and unfolding in BRST first-quantized terms," Commun. Math. Phys. 260 (2005) 147 [hep-th/0406192].

[17] X. Bekaert, "Comments on higher-spin symmetries," Int. J. Geom. Meth. Mod. Phys. 6 (2009) 285 [arXiv:0807.4223 [hep-th]].

[18] M. Grigoriev, "Parent formulations, frame-like Lagrangians, and generalized auxiliary fields," arXiv:1204.1793 [hep-th].

[19] A. Campoleoni, S. Fredenhagen, S. Pfenninger and S. Theisen, "Asymptotic symmetries of three-dimensional gravity coupled to higher-spin fields," JHEP 1011 (2010) 007 [arXiv:1008.4744 [hep-th]].

[20] M. P. Blencowe, "A Consistent Interacting Massless Higher Spin Field Theory In D = (2+1)," Class. Quant. Grav. 6 (1989) 443.

[21] E. Bergshoeff, M. P. Blencowe and K. S. Stelle, "Area Preserving Diffeomorphisms And Higher Spin Algebra," Commun. Math. Phys. 128 (1990) 213.

[22] A. Campoleoni, "Higher Spins in D = 2+1," arXiv:1110.5841 [hep-th].

[23] A. Campoleoni, S. Fredenhagen and S. Pfenninger, "Asymptotic W-symmetries in threedimensional higher-spin gauge theories," JHEP 1109 (2011) 113 [arXiv:1107.0290 [hep-th]].

[24] C. Fronsdal, "Massless Fields With Integer Spin," Phys. Rev. D 18 (1978) 3624.

[25] M. Henneaux and S.-J. Rey, "Nonlinear $W_{\infty}$ as Asymptotic Symmetry of ThreeDimensional Higher Spin Anti-de Sitter Gravity," JHEP 1012 (2010) 007 [arXiv:1008.4579 [hep-th]].

[26] M. R. Gaberdiel and T. Hartman, "Symmetries of Holographic Minimal Models," JHEP 1105 (2011) 031 [arXiv:1101.2910 [hep-th]].

[27] S. F. Prokushkin and M. A. Vasiliev, "Higher spin gauge interactions for massive matter fields in 3-D AdS space-time," Nucl. Phys. B 545 (1999) 385 [hep-th/9806236].

[28] M. R. Gaberdiel and R. Gopakumar, "An $A d S_{3}$ Dual for Minimal Model CFTs," Phys. Rev. D 83 (2011) 066007 [arXiv:1011.2986 [hep-th]]. 
[29] M. R. Gaberdiel and R. Gopakumar, "Minimal Model Holography," arXiv:1207.6697 [hepth].

[30] M. Bañados, C. Teitelboim and J. Zanelli, "The Black hole in three-dimensional spacetime," Phys. Rev. Lett. 69 (1992) 1849 [hep-th/9204099].

[31] M. Bañados, M. Henneaux, C. Teitelboim and J. Zanelli, "Geometry of the $(2+1)$ black hole," Phys. Rev. D 48 (1993) 1506 [gr-qc/9302012].

[32] M. Gutperle and P. Kraus, "Higher Spin Black Holes," JHEP 1105 (2011) 022 [arXiv:1103.4304 [hep-th]].

[33] M. Ammon, M. Gutperle, P. Kraus and E. Perlmutter, "Spacetime Geometry in Higher Spin Gravity," JHEP 1110 (2011) 053 [arXiv:1106.4788 [hep-th]].

[34] P. Kraus and E. Perlmutter, "Partition functions of higher spin black holes and their CFT duals," JHEP 1111 (2011) 061 [arXiv:1108.2567 [hep-th]].

[35] A. Castro, E. Hijano, A. Lepage-Jutier and A. Maloney, "Black Holes and Singularity Resolution in Higher Spin Gravity," JHEP 1201 (2012) 031 [arXiv:1110.4117 [hep-th]].

[36] M. Bañados, R. Canto and S. Theisen, "The Action for higher spin black holes in three dimensions," JHEP 1207 (2012) 147 [arXiv:1204.5105 [hep-th]].

[37] R. M. Wald, "Black hole entropy is the Noether charge," Phys. Rev. D 48 (1993) 3427 [gr-qc/9307038].

[38] C. Aragone and S. Deser, "Consistency Problems Of Spin-2 Gravity Coupling," Nuovo Cim. B 57 (1980) 33.

[39] E. S. Fradkin and M. A. Vasiliev, "Cubic Interaction in Extended Theories of Massless Higher Spin Fields," Nucl. Phys. B 291 (1987) 141.

[40] N. Boulanger, S. Leclercq and P. Sundell, "On The Uniqueness of Minimal Coupling in Higher-Spin Gauge Theory," JHEP 0808 (2008) 056 [arXiv:0805.2764 [hep-th]].

[41] C. Fronsdal, "Singletons And Massless, Integral Spin Fields On De Sitter Space (Elementary Particles In A Curved Space. 7)," Phys. Rev. D 20 (1979) 848.

[42] E. Witten, "(2+1)-Dimensional Gravity as an Exactly Soluble System," Nucl. Phys. B 311 (1988) 46.

[43] M. R. Gaberdiel, T. Hartman and K. Jin, "Higher Spin Black Holes from CFT," JHEP 1204 (2012) 103 [arXiv:1203.0015 [hep-th]].

[44] A. Perez, D. Tempo and R. Troncoso, "Higher spin gravity in 3D: black holes, global charges and thermodynamics," arXiv:1207.2844 [hep-th].

[45] B. de Wit and D. Z. Freedman, "Systematics of Higher Spin Gauge Fields," Phys. Rev. D 21 (1980) 358. 
[46] T. Damour and S. Deser, "'Geometry' Of Spin 3 Gauge Theories," Ann. Inst. Henri Poincaré (A) 47 (1987) 277.

[47] D. Francia and A. Sagnotti, "Free geometric equations for higher spins," Phys. Lett. B 543 (2002) 303 [hep-th/0207002].

[48] D. Francia, J. Mourad and A. Sagnotti, "Current Exchanges and Unconstrained Higher Spins," Nucl. Phys. B 773 (2007) 203 [hep-th/0701163].

[49] D. Francia, "On the Relation between Local and Geometric Lagrangians for Higher spins," J. Phys. Conf. Ser. 222 (2010) 012002 [arXiv:1001.3854 [hep-th]].

[50] J. M. Martin-Garcia, xAct: Efficient tensor computer algebra for Mathematica, http://xact.es/

[51] I. L. Buchbinder, T. V. Snegirev and Yu. M. Zinoviev, "On gravitational interactions for massive higher spins in $A d S_{3}, "$ arXiv:1208.0183 [hep-th]. 